\title{
LITHOGEOCHEMISTRY OF PALEOPROTEROZOIC ORTHOGRANULITES FROM THE RIO PRETO (MG)-VASSOURAS (RJ) REGION, CENTRAL RIBEIRA BELT, SE BRAZIL
}

\author{
MONICA HEILBRON*, RÔMULO MACHADO** \& MARIO FIGUEIREDO** (in memorian)
}

\begin{abstract}
RESUMO LITOGEOQUÍMICA DOS ORTOGRANULITOS DO COMPLEXO JUIZ DE FORA NA SEÇÃO TRANSVERSAL BOM JARDIM DE MINAS (MG)-VASSOURAS (RJ), SEGMENTO CENTRAL DA FAIXA RIBEIRA As rochas granulíticas do Complexo Juiz de Fora integram o embasamento pré-1.8 Ga da escama tectônica Média do segmento Central da Faixa Ribeira. Este domínio é marcado pela interdigitação tectônica de granulitos com unidades metassedimentares meso a neoproterozóicas. O Complexo Juiz de Fora é representado, na região entre Rio Preto (MG) e Vassouras (RJ), por três conjuntos litológicos: ortogranulitos, ortognaisses e paragranulitos, que afloram em diversas escamas de empurrão relacionadas ao encurtamento da Orogênese Brasiliana. Os contatos tectônicos principais são caracterizados pela transformação dos granulitos em milonitos retrometamórficos para a fácies anfibolito. A investigação geoquímica dos ortogranulitos permitiu subdividi-los em duas associações tectônicas distintas: a) uma representada por duas séries cálcio-alcalinas geoquimicamente semelhantes às de arcos magmáticos modernos; b) outra com dois grupos de rochas básicas, urn toleítico e outro com basaltos transicionais a alcalinos, ambos indicando ambiente distensivo intracontinental. A relação entre estes ambientes tectônicos distintos deve ser investigada em termos geocronológicos, já que a tectônica Brasiliana obliterou a paleogeografia deste importante segmento paleoproterozóico da faixa Ribeira.
\end{abstract}

Palavras chave: embasamento paleoproterozóico, margem continental ativa, magmatismo intraplaca, granulitos, Complexo Juiz de Fora

\begin{abstract}
The granulites of the Juiz de Fora Complex (JFC) integrate the pre-1.8 Ga basement of the Intermediate Thrust Sheet of the Ribeira Belt's central segment. This tectonic domain is characterized by intense tectonic imbrication of the JFC granulites with Meso- to Neoproterozoic metasedimentary units. In the Rio Preto (MG)-Vassouras (RJ) region, the JFC comprises three lithological associations: orthogranulites, orthogneisses and paragranulites which crop out as several thrust sheets formed by intense shortening during the Brasiliano Orogeny. The main contacts are characterized by the transformation of the granulites into amphibolite facies mylonites. The geochemistry of the orthogranulites leads to the identification of two tectonic associations. One comprises two calc-alkaline series compositionally similar to rocks of modern magmatic arcs, while the other contains two groups of basic rocks, respectively the tholeiitic series and alkaline to transitional basalts, both of intraplate extensional tectonic setting. The relationships between these distinct tectonic environments need further geochronological background, as the Brasiliano shortening obliterated the original paleogeography of this important Paleoproterozoic segment of the Ribeira belt.
\end{abstract}

Keywords: Paleoproterozoic basement, Continental active margin; Continental intraplate magmatism, Granulites, Juiz de Fora Complex

\section{INTRODUCTION AND TECTONIC SETTING}

The geochemical investigation of high grade metamorphic complexes, such as the Juiz de Fora Complex (JFC), provides key information on protoliths and their tectonic setting, as the primary textures and original relationships between different rock associations were destroyed by the intense deformation and high temperature metamorphism. Geologic investigation of granulite terranes also provides important inferences on the composition and processes of deeper segments of the continental crust. This paper presents and discusses geological and geochemical data from granulites of the JFC, an important segment of the pre-1.8 Ga basement of the Central Segment of Ribeira Belt (Fig. 1) that was reworked during the Brasiliano Orogeny.

The term Juiz de Fora Series (Ebert 1957, 1968, Cordani et al 1973) was originally used to designate metasedimentderived granulites exposed in the central segment of Ribeira belt. Later, orthogranulites were also included in the JFC (Barbosa \& Sad 1983a, b, Machado Filho et al. 1983, Oliveira $1980)$. $\mathrm{U} / \mathrm{Pb}$ and $\mathrm{Rb} / \mathrm{Sr}$ isotopic data indicate that the granulite facies metamorphism took place during the Paleoproterozoic Transamazonian Orogeny, further retrograded to amphibolite facies during the Neoproterozoic Brasiliano Orogeny (Cordani et al. 1973, Machado Filho et al 1983, Teixeira \& Figueiredo 1991). The suggestion that the JFC protoliths may be Archaean (Fyfe \& Leonardos 1974, Cordani et al, in
Fonseca et al 1979, Oliveira 1980) completes the evolution scheme proposed for this complex.

\section{GEOLOGICAL CONTEXT OF JUIZ DE FORA COMPLEX IN THE INVESTIGATED TRANSECT}

Detailed geologic mapping in the Bom Jardim de Minas (MG)-Piraí (RJ) Rio Preto (MG)-Vassouras (RJ) section allowed the subdivision of the JFC into four lithologic units (Heilbron 1993): (a) orthogranulites, with amphibolite facies retrogressive mylonitic parageneses; (b) banded orthogneisses of amphibolite facies; (c) metasedimentary granulites (kinzigites with minor orthopyroxene in leucossomes); and (d) amphibolite facies metasedimentary gneisses. In this paper and based on structural-metamorphic data combined with new U/Pb geochronological data (Machado et al 1996), we assume that the paragneisses represent Meso to Neoproterozoic units, correlated to the Andrelândia Depositional Cycle (Andreis et al 1989). They were technically imbricated with the Paleoproterozoic orthogranulites and orthogneisses of the JFC during the Brasiliano orogeny. Thus, the JFC comprises the granulite facies basement (Pre-1,8 Ga) of the central segment of Ribeira belt. One U/Pb discordia (Machado et al. 1996) and $\mathrm{Rb} / \mathrm{Sr}$ errorchrons (Heilbron 1993) support the Paleoproterozoic age of the granulite facies metamorphism in the investigated region.

* Departamento de Geologia Regional e Geotectônica, Faculdade de Geologia, Universidade do Estado do Rio de Janeiro. Rua São Francisco Xavier 524, sala A-4024, Maracana, Rio de Janeiro. CEP 20559-900. Fax-021-2546675. Tel- 021-5877102 - e-mail: heilbron@uerj.br

** Institute de Geociências, Universidade de São Paulo, Caixa Postal 11348, São Paulo, SP, CEP 05422-970 


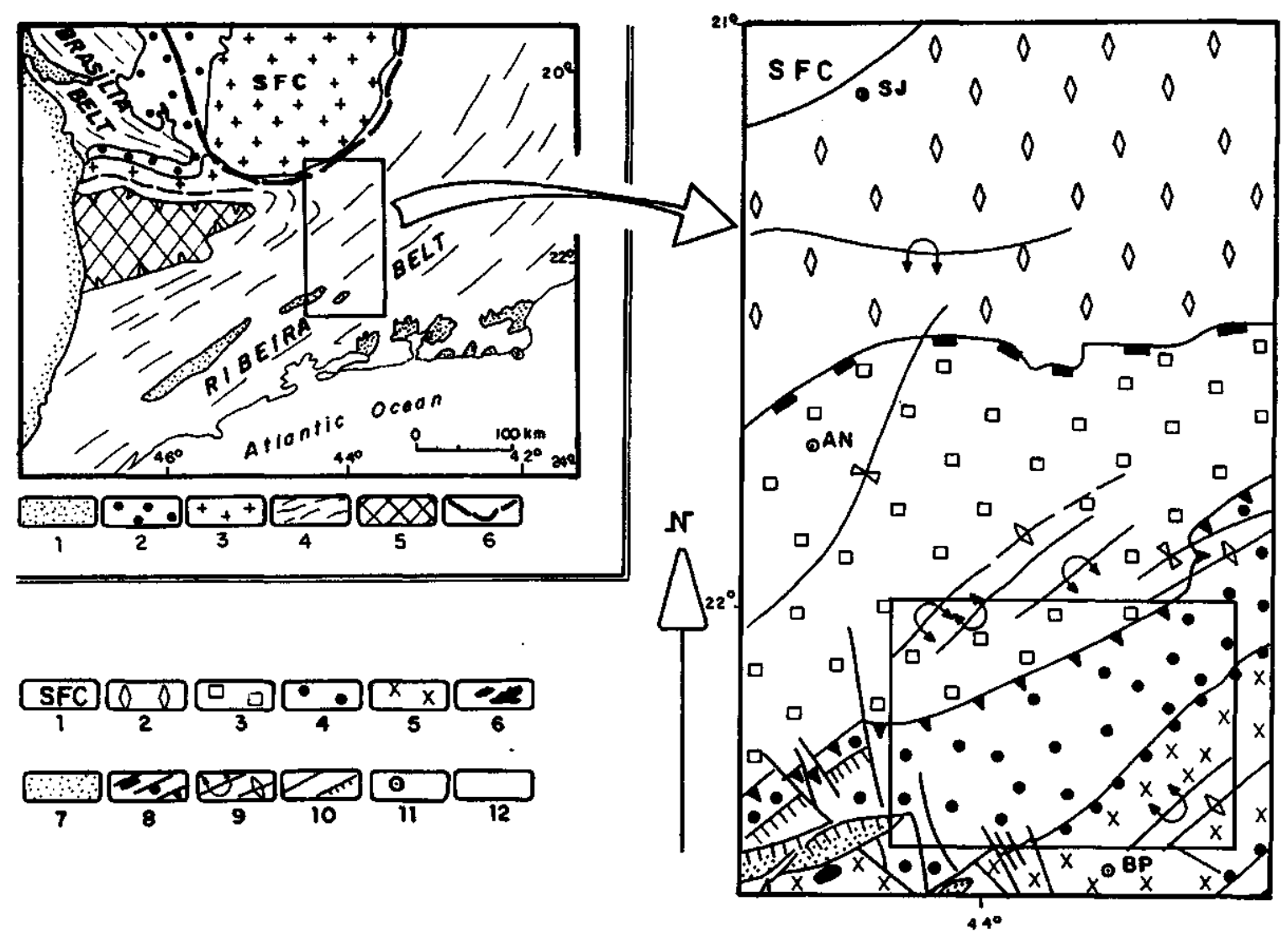

Figure 1 - (a) - Simplified tectonic map of southeastern Brazil, compiled and integrated from Hasui \& Oliveira (1984), Campos Neto (1992), Valeriano (1992), and Trouw \& Castro (1996). The inset shows the location of the central segment of Ribeira Belt. Legend: 1-Phanerozoic cover, a -Parana basin; b, c, $d$ and e - Taubate, Resende, Volta Redonda and Guanabara rifts; 2- Bambui Group, 3 -cratonic basement, 4 -Brasiliano fold belts (ARGD-Alto Rio Grande Domain); 5 - Guaxupe nappe; 6 - Limit of the São Francisco Craton.(b) - Tectonic compartments of the central segment of Ribeira Fold Belt (Heilbron 1993,1995). Legend: 1- São Francisco craton; 2 - autochthonous domain; 3 - lower domain; 4 - intermediate domain; 5 - upper domain; 6 - Meso-Cenozoic alkaline rocks; 7 - Phanerozoic cover; 8 -Major thrust shear zones; 9 - Major late deformation axial traces; 10 - brittle phanerozoic faults; 11 - Towns (SJ-São Joao del Rei, AN-Andrelândia, BP-Barra do Pirai); 12-Location of the investigated transect of figure 2.

Figure 1 - (a) - Mapa tectonico simplificado da região sudeste brasileira, compilado e integrado de Hasui \& Oliveira (1984), Campos Neto (1992), Valeriano (1992) e Trouw \& Castro. (1996). O paralelogramo localiza o segmento central da Faixa Ribeira. Legenda: 1 - Cobertura fanerozóica, a - Bacia do Paraná; de d a f, respectivamente, rifts de Taubaté, Resende, Volta Redonda e da Guanabara; 2 - Grupo Bambuí; 3 - Embasamento cratônico; 4 - Faixas dobradas brasilianas (ARGD - Domínio Alto Rio Grande); 5 - Nappe Guaxupé; 6 - Limite do Cráton do São Francisco, (b) - Compartimentacão tectônica do segmento central da Faixa Ribeira (Heilbron 1993,1995). Legenda: 1 -Cráton do São Francisco; 2 - Domínio Autóctone; 3 - Domínio Inferior; 4 - Domínio Médio; 5 - Domínio Superior; 6 -Rochas alcalinas meso-cenozóicas; 7 - Cobertura fanerozóica; 8 - Principais empurrões; 9 - Principais traços axiais do redobramento tardio; 10 - Falhas rúpteis fanerozóicas, 11 -Cidades (SJ- São Jão del Rei, NA - Andrelândia, BP - Barra do Piraí), 12 - Área investigada em detalhe, apresentada na figura 2.

Figure 2 shows that the orthogranulites of the JFC crop out as three major tectonic thrust sheets. Their contacts are important shear zones, characterized by retrogressive amphibolite facies mylonitic textures and tectonic mixing with the Meso to Neoproterozoic cover. Within the thrust sheets, coarse granoblastic textures with green feldspars predominate.

Along the transect, granulites with intermediate composition are the most abundant rocks of the JFC, but many visited outcrops show acidic and basic types, except at the basal section of the upper granulite thrust sheet (Fig. 2), which consists of basic tholeiitic rocks. Locally, granulites display relict migmatitic structures.

Ortho and clinopyroxene, hornblende, quartz and antiperthitic plagioclase are major minerals of the orthogranulites, with minor garnet and biotite. K-feldspar is restricted to the acid terms (Table 1). Zircon, apatite, monazite, and ilmenite are accessories. Retrogressive reactions, such as hornblende and biotite rims and lamellae around and within the pyroxene crystals are common, mainly along major Brasiliano shear zones, where the granulites are transformed into banded mylonite-gneisses.

The JFC orthogneisses (Fig. 2) are deformed acid to basic igneous rocks metamorphosed under amphibolite facies. The geochemistry of these orthogneisses will not be considered in this paper, but they probably represent the igneous protoliths of the granulites.

The paragranulites of the JFC (Fig. 2) are kinzigites with orthopyroxene-bearing leucossomes. They also occur in the studied transect, but are restricted to the basal thrust sheet (Fig. 2). Eastwards, nearby the city of Juiz de Fora, the paragranulites of the JFC become more abundant (Duarte et al. 1994). The kinzigites are made up of garnet, sillimanite, biotite, plagioclase, quartz, and minor K-feldspar and orthopyroxene.

GEOCHEMISTRY Previous results Oliveira $(1980,1982)$ presented geochemical data of the JFC rocks and concluded that these rocks belong to differentiated calc- 


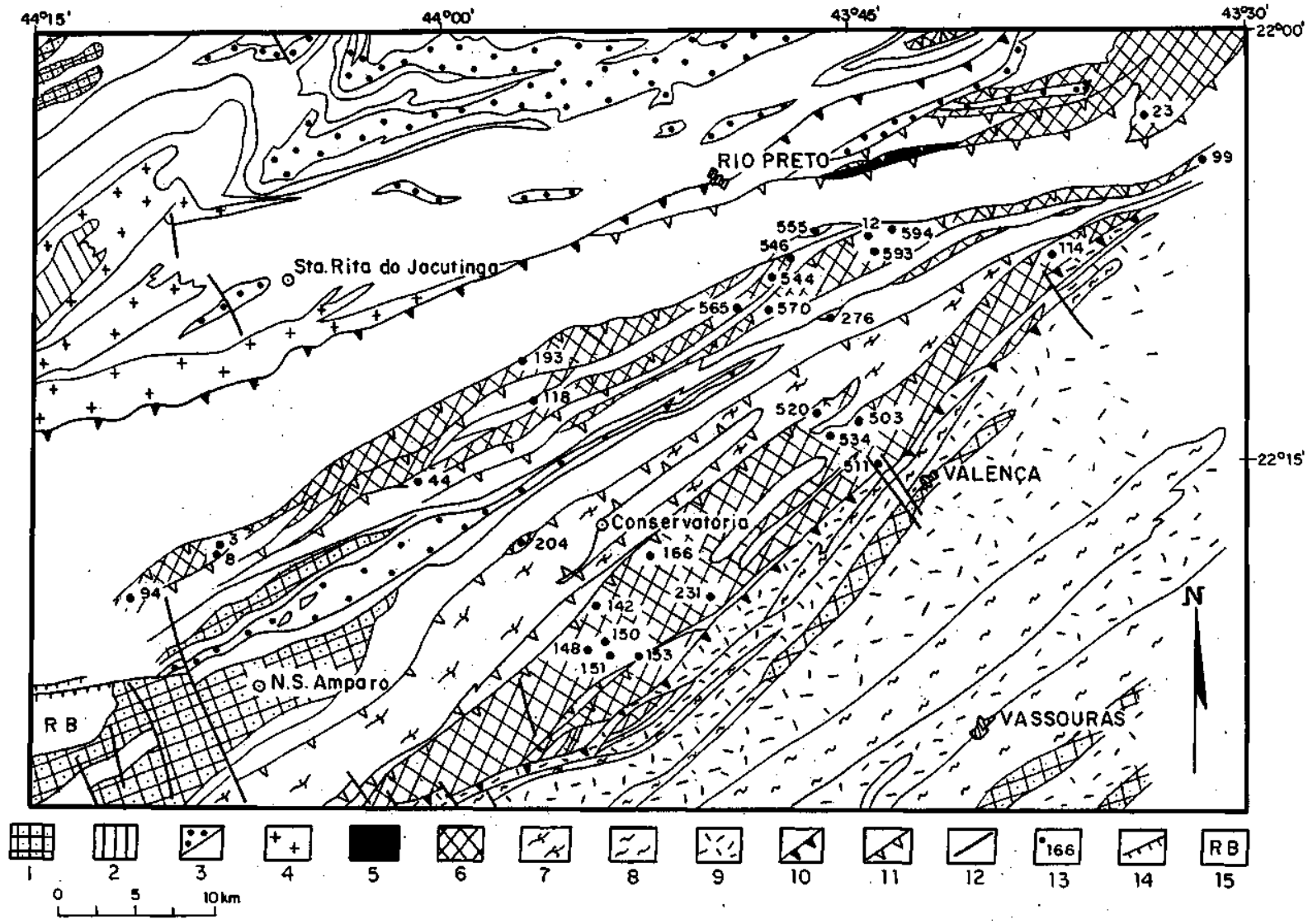

Figure 2 - Detailed geological map of the Rio Preto (MG) - Vassouras (RJ) region, with the location of the analysed granulite samples. Legend: 1 - S-type Brasiliano granitoids; 2 - I-type Brasiliano granitoids; 3 - Andreldndia depositional cycle discriminating major quartzite layers; 4 - Mantiqueira complex; 5 - Paragranulites of JFC (?); 6 - Orthogranulites of JFC; 1 Hornblende orthogneisses of JFC; 8 - Paraiba do Sul group or complex; 9 - Quirino complex; 10 - Major thrust shear- zones limiting the Lower, Intermediate and Upper tectonic domains; 11 - Subordinated thrusts; 12 - Subvertical faults; 13 - Sample location; 14 - Normal faults; 15 - Resende basin.

Figura 2 - Mapa geológico detalhado da Região entre Rio Preto(MG) e Vassouras (RJ), com a localização das amostras estudadas. Legenda - 1 -Granitóides brasilianos tipo-S; 2 - Granitóides brasilianos Tipo-I; 3 -Ciclo Deposicional Andrelândia com bancos quartzíticos individualizados; 4 - Complexo Mantiqueira; 5 -Paragranulitos do CJF (?); 6 - Ortogranulitos do CJF; 7 -Hornblenda ortognaisses do CJF; 8 - Complexo Paraíba do Sul; 9 - Complexo Quirino; 10 - Principais empurrões basais dos domímios tectônicos Médio e Superior; 11 - Empurrões subordinados; 12 - Falhas subverticais; 13 - Localização das amostras; 14 - Falhas normais e 15 - Bacia de Resende

alkaline series, with depletion of $\mathrm{Rb}$ and slight enrichment of $\mathrm{Zr}$, Sr and $\mathrm{Ba}$ as compared to the average upper crust composition. The author also proposed that the granulites represent an alkali depleted residue of extensive anatexis.

Machado Filho et al. (1983) subdivided the JFC into orthoand paragranulites, the former belonging both to the calcalkaline and tholeiitic series. The calc-alkaline orthogranulites was intepreted as representing lower crust samples, while the tholeiitic granulites were correlated to an Archean basaltic crust. The calc-alkaline and tholeiitic nature of the JFC granulites were also recognized by Barbosa \& Sad (1983 a, b) and Sad \& Barbosa (1985). The authors suggest that the calc-alkaline granulites may represent either metasedimentary rocks or injection of a acid magma within the basic rocks, while the tholeiitic samples characterize a mafic oceanic crust.

Figueiredo \& Campos Neto (1993) and Campos Neto \& Figueiredo (1990) discuss geochemical data of the northern segment of the JFC in the Rio de Janeiro state, and describe the occurrence of Orthogranulites (Itaperuna-Comendador Venancio unit) and amphibolite facies orthogneisses (Sao João do Paraíso unit) with calc-silicate inclusions. The analytical data of both units are similar and indicate two calc- alkaline series. One is represented by a large compositional variety of rocks, enriched in $\mathrm{Mg}, \mathrm{Ca}$ and $\mathrm{Cr}$, and with slightly fractionated chondrite REE normalized patterns. The other is enriched in LILE, with higher Fe, Ti, K, Rb, Ba, Nb, Zr and HREE contents, and more fractionated chondrite normalized REE patterns with negative Eu anomaly.

Sampling Based on field and petrographic data (Table 1), 37 samples of granulites were selected for chemical analyses. The location of the sampled outcrops is shown in figure 2. The analyses were performed at Geosol-Geolab Division Laboratory, Brazil. Si, Al, Ti, Ca, Fe ${ }^{\text {tot }}, \mathrm{P}$, and trace elements were analysed by XRF, while $\mathrm{Mn}, \mathrm{Ca}, \mathrm{Na}, \mathrm{K}$, and $\mathrm{Mg}$ by atomic absorption, $\mathrm{Fe}^{2+}$ by wet chemical analysis, and REE by fusion-ICP. The analytical results are in Tables 2 to 4 .

Chemical discrimination and tectonic settings The studied granulites subdivide into two groups of acid to intermediate composition (Table 2 and 3) and two groups of basic composition (Table 4).

ACID TO INTERMEDIATE GRANULITES These granulites comprise two geochemical groups, named CAS1 
Table 1- Simplified mineralogy of the granulites of the Juiz de Fora Complex, subdivided into distinctive geochemical groups. Mineral abbreviations: OPX- orthopyroxene; CPX-clinopyroxene; HBL-hornblende; BIO- biotite; PLAG-plagiodase; QZquartz; KF-alcali-feldspar; ZIR-zircon; AP-apatite; GAR-garnet; OP-opaque minerals

Tabela 1- Mineralogia simplicada dos granulitos do Complexo Juiz de Fora, subdivididos pelos diferentes grupos geoquímicos.

\begin{tabular}{|c|l|c|l|}
\hline Group & \multicolumn{1}{|c|}{ Sample } & Rock type & \multicolumn{1}{|c|}{ Mineralogy } \\
\hline \multirow{3}{*}{ CAS1 } & $\begin{array}{l}\text { BP-CM 150e; RPM-555; BP-CM-153b; } \\
\text { VAL-M-276; BP-CM-231; NSA-5-9a; } \\
\text { BP-CM-142; VAL-I-12; NSA-5-8; VAL-IV-23 }\end{array}$ & Intermediate & OPX; HBL; BIO; PLAG; QZ; ZIR; AP; OP \\
\cline { 2 - 4 } & RPM-546; RPM-193e; VAL-593 & Acid & OPX; HBL; BIO; PLAG; QZ; KF; ZIR; OP \\
\hline \multirow{2}{*}{ CAS22 } & VAL-511a; BP-CM-148; VAL-III-11 & Intermediate & OPX \\
\cline { 2 - 4 } & $\begin{array}{l}\text { RPM-344e; RPM-565a; RPM-570; BP-CM-150a; } \\
\text { NSA-5-9c; CJE-44-7; VAL-IV-99 }\end{array}$ & Acid & OPX; BIO; KF; PLAG; QZ; ZIR; OP; GAR \\
\hline \multirow{3}{*}{ B1 } & CPH-118 & Ultrabasic & CPX; HBL; OP; PLAG \\
\cline { 2 - 4 } & $\begin{array}{ll}\text { NSA-CM-94; BP-CM-151; RPM-520b; RPM-534; } \\
\text { VAL-M-503a; VAL-M-503b; BP-CM-166b }\end{array}$ & Basic & CPX; OPX; HBL; BIO; PLAG; QZ; ZIR; AP \\
\hline \multirow{2}{*}{ B2 } & CJE-44-1; CJE-44-11; CON-204; NSA-5-9d & Basic & CPX; OPX; HBL; BIO; PLAG; QZ; ZIR; AP \\
\cline { 2 - 4 } & CJE-44-6b & Intermediate & OPX; PLAG; QZ; ZIR; OP \\
\hline
\end{tabular}

and CAS2, which probably belong to different calc-alkaline series (Figs. 3a and 3b). They are metaluminous rocks with Shand's index below 1 (Fig. 3c). Their chemical composition classification, also corroborated by petrographic data, indicate the predominance of intermediate (enderbitic) rocks, as shown in the Alk x SiO 2 diagram of Le Maitre (1989) and in the Middlemost (1985) diagram (Figs. 3d and 3e), and by the QAP normative diagram (Fig. 3f).

The CAS1 samples belong to a medium-K calc-alkaline series (open circles in all diagrams). It is the most expanded series and includes gabbrodiorite, diorite, tonalite, granodiorite and one granite sample (Figs. 3d to 3f)- As compared to the CAS2 series (open squares), discussed below, the CAS1 is relatively enriched in compatible elements $(\mathrm{CaO}$, $\mathrm{MgO}, \mathrm{FeO}^{*}, \mathrm{MnO}, \mathrm{Cr}$ ), and HFS elements (e.g. $\mathrm{TiO}_{2}, \mathrm{P}_{2} \mathrm{O}_{5}$ ), as shown respectively in figures $4 \mathrm{a}$ and $4 \mathrm{~b}$. In spite of the LILE scatter relative to $\mathrm{SiO}_{2}$ (Fig. 4c), probably due to metamorphic remobilization during the Brasiliano Cycle, the CAS1 samples generally have lower contents in these elements. REE normalized to chondrite are more enriched but less fractionated then the CAS2 (Figs. 5a, 5b), show large negative $\mathrm{Eu}$ anomalies $\left(\mathrm{Eu} / \mathrm{Eu}^{*}\right.$ between 0.35 and 0.80$)$, and the HREE content decreases with differentiation (Fig. 5a). The CAS1 rocks also show the lowest $\mathrm{Rb} / \mathrm{Sr}$ and the highest $\mathrm{Sm} / \mathrm{Nd}$ ratios. Two samples (VAL-IV-23, VAL-593), represented by half-filled circles in the Harker diagrams (Figs. 4a to 4c), have some chemical similarities with CAS 1, but their chondrite normalized REE pattern is more fractionated, lack negative $\mathrm{Eu}$ anomaly $\left(\mathrm{Eu} / \mathrm{Eu}^{*} \approx 1\right)$, and have higher $\mathrm{Cr}$ contents.

The CAS2 samples (open squares) belong to a high-K calc-alkaline series, with granites, and minor granodiorites and monzodiorites, containing up to $4.1 \% \mathrm{~K}_{2} \mathrm{O}$ (Fig. $3 \mathrm{~d}$ to $3 \mathrm{f})$. As compared to the CAS1, the CAS2 samples are depleted in HFSE and particularly in compatible elements, except Ni (Fig. 4a, b). This group is also enriched in LILE, especially $\mathrm{K}_{2} \mathrm{O}, \mathrm{Rb}$ and $\mathrm{Sr}$ (Fig. 4c), and is richer in $\mathrm{Al}_{2} \mathrm{O}_{3}$. Chondrite-normalized REE abundances display higher fractionation (Fig. 5c), with substantial depletion in HREE and positive $\mathrm{Eu}$ anomalies (Eu/Eu* up to 1.4). This group also has higher $\mathrm{K} / \mathrm{Rb}, \mathrm{Rb} / \mathrm{Sr}, \mathrm{K} / \mathrm{Ba}$, and $\mathrm{La} / \mathrm{Sm}$ ratios, consistent with the LILE enrichment. U/Pb data on zircons from acid granulites of outcrop 44, located near the town of Conservatoria (Fig. 2), define a discordia with intercepts at 2134 and
$579 \mathrm{Ma}$, while monazite from the same sample is concordant at $563 \mathrm{Ma}$ (Machado et al. 1996). Field relationships indicate that CAS2 is intrusive in CAS 1 and B2 basic group (described below), which also integrate the JFC.

Three samples (half-filled squares) show enrichment in LREE (Fig. 5d) and highly fractionated REE chondritenormalized signature. When compared with the rest of CAS2 samples, they also display enrichment in some HFS elements (e.g. $\mathrm{Y}, \mathrm{Zr}, \mathrm{P}_{2} \mathrm{O}_{5}$ ), and in $\mathrm{Ba}$ and $\mathrm{Cr}$, and have very low $\mathrm{Ni}$ contents (Figs. $4 \mathrm{a}, 4 \mathrm{~b}$ and $4 \mathrm{c}$ ). Due to the small number of samples, the distinct chemical composition of three samples might be due either to a distinct calc-alkaline group or to magmatic differentiation.

The plot of the CAS1 and CAS2 samples in tectonic discrimination diagrams, such as $\mathrm{Rb} \times \mathrm{Y}+\mathrm{Nb}$ (Pearce et al. 1984) suggest a volcanic arc setting (Fig. 6). The R1 x R2 (Batchelor \& Bowden 1985) diagram suggests that the CAS1 rocks may have been derived from pre-collisional granitoids, while the CAS 2 rocks represent late- to post-collisional granitoids (Fig. 7). The $\mathrm{K}_{2} \mathrm{O} \times \mathrm{SiO}_{2}$ diagram (le Maitre 1989) and the $\log \mathrm{CaO} / \mathrm{Alk} \times \mathrm{SiO}_{2}$ diagram (Brown 1982), not shown in this paper, are also suggestive of a progressive chemical maturity from the CAS1 to the CAS2 group, as observed in modern compressive settings, with enrichment in Alk and in the total LILE. The multielemental diagrams such as the Ocean Ridge Granite normalized spidergrams (Fig. 8) of Pearce et al. (1984), are also consistent with magmatic arc settings.

Sample CJE-44-6b (asterisk) is a leucossome associated with alkaline to transitional basic rocks of B2 group (see below). As the other samples of this group, it shows very high contents of HFS elements.

BASIC GRANULITES The basic rocks of the JFC (Table 4) show some compositional dispersion, even within a small number of samples. They are tentatively subdivided in two groups.

The $\mathbf{B l}$ group (triangles) is characterized by low-TiO2 $\mathrm{P}_{2} \mathrm{O}_{5}$ tholeiitic basic rocks (Figs. 9 to 12 ) and one ultrabasic sample (CPH-118). The basic samples classify as olivine gabbros, norites, and gabbro-diorites in the R1R2 diagram (not shown). The chemical classification of Jensen (1976) (Fig. 11) and the binary variation diagrams (Figs. 12a and $12 \mathrm{~b}$ ) indicate that two of the tholeiitic basic samples (inverted 
Table 2- Geochemical data of acid to intermediate granulites of JFC: Data of CAS1 group. Underlined values represent the detection limit of the laboratory

Tabela 2- Dados geoquímicos de granulitos ácidos a intermediários do JFC: Análises do grupo CASl. Os valores sublinhados representam o limite de detecção do laboratório.

\begin{tabular}{|c|c|c|c|c|c|c|c|c|c|c|c|c|c|}
\hline \multirow[b]{2}{*}{ Sample } & \multicolumn{11}{|c|}{ CAS1 } & \multirow[b]{2}{*}{$\underset{23}{\text { VAL IV }}$} & \multirow[b]{2}{*}{$\begin{array}{c}\text { VAL } \\
593\end{array}$} \\
\hline & $\begin{array}{l}\text { BP-CM- } \\
150 E\end{array}$ & $\underset{555}{\text { RPM: }}$ & $\begin{array}{c}\text { BP-CM- } \\
\text { 153B }\end{array}$ & $\underset{276}{\text { VAL-M- }}$ & $\begin{array}{c}\text { BP-CM- } \\
231\end{array}$ & $\begin{array}{c}\text { NSA-5- } \\
9 A\end{array}$ & $\begin{array}{c}\text { BP-CM- } \\
142\end{array}$ & $\begin{array}{l}\text { VALI } \\
12\end{array}$ & NSA-5-8 & $\begin{array}{c}\text { RPM- } \\
546\end{array}$ & $\begin{array}{l}\text { RPM- } \\
\text { 193E }\end{array}$ & & \\
\hline $\mathrm{SiO}_{2}$ & 57.10 & 57.60 & 57.90 & 58.00 & 58.80 & 59.10 & 61.80 & 61.60 & 61.30 & 62.90 & 65.10 & 52.30 & 66.80 \\
\hline $\mathrm{TiO}_{2}$ & 0.58 & 0.83 & 0.89 & 0.87 & 0.75 & 0.86 & 1.00 & 0.65 & 0.70 & 0.83 & 0.45 & 0.92 & 0.34 \\
\hline $\mathrm{Al}_{2} \mathrm{O}_{3}$ & 14.30 & 15.80 & 16.70 & 15.80 & 15.70 & 15.80 & 15.20 & 15.30 & 17.00 & 14.70 & 14.80 & 16.00 & 14.80 \\
\hline $\mathrm{Fe}_{2} \mathrm{O}_{3}$ & 0.98 & 2.10 & 1.10 & 5.00 & 3.30 & 1.40 & 2.00 & 2.96 & 0.58 & 1.50 & 1.10 & 3.36 & 1.40 \\
\hline $\mathrm{FeO}$ & 7.40 & 6.60 & 7.00 & 3.20 & 4.60 & 5.80 & 5.20 & 4.40 & 5.20 & 5.00 & 3.10 & 6.70 & 3.00 \\
\hline $\mathrm{MnO}$ & 0.22 & 0.25 & 0.18 & 0.15 & 0.15 & 0.15 & 0.15 & 0.16 & 0.15 & 0.11 & 0.08 & 0.19 & 0.11 \\
\hline $\mathrm{MgO}$ & 5.70 & 3.90 & 3.40 & 3.60 & 3.30 & 3.60 & 1.80 & 3.50 & 2.90 & 2.50 & 2.40 & 7.40 & 2.30 \\
\hline $\mathrm{CaO}$ & 7.90 & 5.00 & 6.20 & 6.30 & 6.10 & 5.30 & 4.80 & 4.90 & 5.10 & 3.90 & 2.70 & 7.40 & 4.00 \\
\hline $\mathrm{Na}_{2} \mathrm{O}$ & 4.20 & 3.80 & 4.50 & 4.00 & 4,30 & 4.40 & 5.00 & 3.36 & 3.50 & 4.20 & 3.20 & 2.90 & 3.50 \\
\hline $\mathrm{K}_{2} \mathrm{O}$ & 0.38 & 2.20 & 1.40 & 1.80 & 1.70 & 2.10 & 1.20 & 1.60 & 1.80 & 2.00 & 4.70 & 1.30 & 2.80 \\
\hline $\mathrm{P}_{2} \mathrm{O}_{5}$ & 0.05 & 0.61 & 0.33 & 0.20 & 0.24 & 0.32 & 0.33 & 0.16 & 0.30 & 0.20 & 0.14 & 0.15 & 0.12 \\
\hline $\mathrm{H}_{2} \mathrm{O}$ & 0.45 & 0.73 & 0.41 & 0.51 & 0.47 & 0.63 & 0.54 & - & 0.81 & 0.74 & 0.70 & $\cdot$ & - \\
\hline $\mathrm{CO}_{2}$ & 0.55 & 0.40 & 0.25 & 0.25 & 0.25 & 0.40 & 0.80 & $=$ & 0.40 & 1.00 & 1.30 & . & - \\
\hline LOI & 0.22 & 0.01 & 0.11 & 0.03 & 0.03 & 0.14 & 0.16 & 0.52 & 0.10 & 0.04 & 0.09 & 0.01 & 0.29 \\
\hline Total & 100.03 & 99.83 & 100.37 & 99.71 & 99.69 & 99.37 & 99.98 & 99.11 & 99.84 & 99.62 & 99.86 & 98.63 & 99.46 \\
\hline $\mathbf{M g} \#$ & 55.09 & 45.01 & 43.13 & 45.45 & 43.72 & 47.61 & 31.42 & 46.89 & 47.46 & 41.23 & 51.12 & 57.56 & 49.04 \\
\hline $\mathrm{Cr}$ & 120 & . & 79 & - & - & 100 & 23 & 109 & 59 & - & - & 342 & 68 \\
\hline $\mathbf{N i}$ & 71 & - & 56 & - & - & 62 & 55 & 24 & 55 & - & - & 41 & 24 \\
\hline Co & - & 46 & - & 87 & 51 & - & - & - & - & 41 & 46 & - & - \\
\hline $\mathrm{V}$ & - & 98 & - & 120 & 130 & - & - & - & - & 130 & 72 & - & - \\
\hline $\mathrm{K}$ & 3154 & 18263 & 11622 & 14942 & 14112 & 17433 & 9962 & - & 14942 & 16603 & 39016 & - & - \\
\hline $\mathbf{R b}$ & 12 & 94 & 29 & 15 & 34 & 87 & 18 & 77 & 91 & 100 & 190 & 41 & 70 \\
\hline $\mathrm{Cs}$ & - & - & $=$ & $=$ & $=$ & - & * & 5.00 & - & - & - & 5.00 & 5.00 \\
\hline $\mathrm{Ba}$ & 280 & 358 & 620 & 421 & 1254 & 880 & 730 & 389 & 660 & 546 & 896 & 403 & 2006 \\
\hline Sr & 270 & 290 & 600 & 300 & 600 & 530 & 490 & 390 & 500 & 350 & 350 & 236 & 506 \\
\hline $\mathrm{Ta}$ & - & - & $=$ & $=$ & $=$ & - & - & 5.00 & - & - & . & 5.00 & 11.00 \\
\hline $\mathrm{Nb}$ & 10 & 17 & 12 & 10 & 12 & 18 & 10 & 8 & 12 & 13 & 19 & 5 & 8 \\
\hline Hf & . & - & $=$ & - & - & - & - & 8.00 & - & $=$ & - & 8.00 & 8.00 \\
\hline $\mathbf{z r}$ & 47 & 240 & 190 & 75 & 180 & 200 & 120 & 145 & 140 & 190 & 250 & 129 & 170 \\
\hline $\mathrm{Ti}$ & 3477 & 4976 & 5336 & 5216 & 4496 & 5156 & 5995 & - & 4197 & 4976 & 2698 & $=$ & - \\
\hline $\mathbf{Y}$ & $\leq 10$ & 51 & 20 & 42 & 24 & 42 & 14 & 30 & 39 & 22 & 18 & 25 & 22 \\
\hline Th & - & - & - & $=$ & - & - & - & 8.00 & - & $=$ & - & 5.00 & 5.00 \\
\hline $\mathbf{U}$ & - & - & - & $=$ &. & - & - & 10.00 &. & - & - & 12.00 & 12,00 \\
\hline $\mathbf{L a}$ & - & 22.48 & - & 31.40 & 23.38 & - & - & 76.87 & - & 38.21 & 26.85 & 18.38 & 15.69 \\
\hline $\mathrm{Ce}$ & - & 60.28 & - & 75.26 & 55.50 & - & - & 136.80 & - & 86.60 & 57.38 & 41.48 & 33.51 \\
\hline Nd & - & 34,08 & - & 35.09 & 27.50 & - & - & 59.27 & - & 35.64 & 24.32 & 15.46 & 12.66 \\
\hline $\mathrm{Sm}$ & - & 7.76 & - & 6.77 & 5.20 & - & - & 9.50 & - & 5.84 & 4.79 & 3.26 & 2.36 \\
\hline $\mathbf{E u}$ & - & 0.89 & . & 1.23 & 1.19 & . & $=$ & 0.99 & $=$ & 0.74 & 0.85 & 0.87 & 0.70 \\
\hline Gd & - & 6.75 & - & 5.09 & 3.86 & - & - & 5.55 & - & 4.30 & 3.61 & 2.47 & 1.90 \\
\hline Dy & - & 6.75 & - & 4.36 & 2.99 & $=$ & - & 4.28 & $=$ & 2.87 & 2.14 & 2.23 & 1.78 \\
\hline Ho & - & 1.48 & - & 0.92 & 0.62 & - & - & 0.80 & $=$ & 0.61 & 0.42 & 0.37 & 0.36 \\
\hline Er & - & 3.92 & - & 2.69 & 1.72 & $=$ & - & 1.84 & - & 1.60 & 1.07 & 0.94 & 0.98 \\
\hline $\mathbf{Y b}$ & - & 3.27 & - & 2.43 & 1.51 & - & - & 1.32 & - & 1.27 & 0.68 & 0.74 & 0.77 \\
\hline Lu & - & 0.42 & - & 0.34 & 0.21 & - & - & 0.15 & - & 0.18 & 0.12 & 0.09 & 0.11 \\
\hline
\end{tabular}


Table 3- Geochemical data of acid to intermediate granulites: Data of CAS2 group (squares symbols). The three samples at the right side of the table belong to the subgroup represented as partial filled squares. Underlined values represent the detection limit of the laboratory

Tabela 3- Dados geoquímicos de granulitos dados a intermediários: Análises do grupo CAS2 (símbolos : quadrados). As tres amostras localizadas no lado direito da tabela pertecem ao subgrupo representado por quadrados parcialmente preenchidos. Os valores sublinhados representam o limite de detecção do laboratório.

\begin{tabular}{|c|c|c|c|c|c|c|c|c|c|c|c|c|}
\hline \multirow[b]{2}{*}{ Sample } & \multicolumn{8}{|c|}{$\operatorname{CSA} 2$} & \multicolumn{3}{|c|}{ CAS2 LREE enriched } & \multirow{2}{*}{$\frac{\text { B2 }}{\underset{\text { CJE-44- }}{\text { 6b }}}$} \\
\hline & $\begin{array}{l}\text { VAL-M- } \\
\text { S11A }\end{array}$ & $\begin{array}{l}\text { RPM- } \\
\text { 344E }\end{array}$ & $\begin{array}{c}\text { RPM- } \\
\text { 565A }\end{array}$ & $\begin{array}{c}\text { RPM- } \\
570\end{array}$ & $\begin{array}{c}\text { BP-CM- } \\
150 \mathrm{~A}\end{array}$ & $\begin{array}{c}\text { NSA-5- } \\
9 B\end{array}$ & $\begin{array}{c}\text { NSA-5- } \\
9 \mathrm{C}\end{array}$ & $\begin{array}{c}\text { CJE-44- } \\
7\end{array}$ & $\begin{array}{c}\text { BP-CM- } \\
148\end{array}$ & $\begin{array}{c}\text { VAL III } \\
11\end{array}$ & $\underset{99}{\text { VAL IV }}$ & \\
\hline $\mathrm{SiO}_{2}$ & 58.10 & 65.00 & 70.50 & 70.70 & 71.00 & 74.60 & 74.70 & 76.20 & 53.50 & 59.60 & 66.50 & 55.70 \\
\hline $\mathrm{TiO}_{2}$ & 0.82 & 0.42 & 0.19 & 0.50 & 0.36 & 0.40 & 0.24 & 0.13 & 1.50 & 0.67 & 0.26 & 2.10 \\
\hline $\mathrm{Al}_{2} \mathrm{O}_{3}$ & 18.10 & 15.60 & 13.90 & 13.90 & 15.40 & 11.80 & 12.60 & 12.00 & 17.20 & 17.00 & 16.40 & 15.40 \\
\hline $\mathrm{Fe}_{2} \mathrm{O}_{3}$ & 1.30 & 1.20 & 0.64 & 0.84 & 0.63 & 0.81 & 0.29 & 0.95 & 1.10 & 2.20 & 1.10 & 3.00 \\
\hline $\mathrm{FeO}$ & 4.80 & 2.90 & 1.40 & 1.40 & 0.87 & 0.98 & 1.01 & 0.14 & 6.80 & 4.60 & 2,00 & 7.10 \\
\hline Mno & 0.09 & 0.06 & 0.04 & 0.04 & 0.05 & 0.05 & 0.05 & 0.02 & 0.22 & 0.10 & 0.01 & 0.22 \\
\hline $\mathrm{MgO}$ & 2.90 & 2.00 & 0.77 & 1.50 & 0.14 & 0.31 & 0.20 & 0.12 & 3.80 & 3.60 & 1.80 & 2.40 \\
\hline $\mathrm{CaO}$ & 5.40 & 2.30 & 2.30 & 2.20 & 1,80 & 1.70 & 1.00 & 1.10 & 5.70 & 3.20 & 2.80 & 5.50 \\
\hline $\mathrm{Na}_{2} \mathrm{O}$ & 5.20 & 3.40 & 3.30 & 3.60 & 4.60 & 3.50 & 3.00 & 3.30 & 4.10 & 3.50 & 4.10 & 5.80 \\
\hline $\mathrm{K}_{2} \mathrm{O}$ & 1.90 & 6.20 & 5.80 & 3.70 & 4.30 & 3.70 & 5.90 & 5.10 & 2.30 & 4.10 & 4.00 & 1.10 \\
\hline $\mathrm{P}_{2} \mathrm{O}_{5}$ & 0.29 & 0.13 & 0.07 & 0.13 & 0.09 & 0.05 & 0.05 & 0.06 & 1.20 & 0.28 & 0.15 & 0.54 \\
\hline $\mathrm{H}_{2} \mathrm{O}$ & 0.55 & 0.24 & 0.28 & 0.48 & 0.42 & 1.21 & 0.59 & 0.42 & 1.56 & $\cdot$ & $\cdot$ & 0.64 \\
\hline $\mathrm{CO}_{2}$ & 0.40 & 0.25 & 0.40 & 0.75 & 0.20 & 0.80 & 0.25 & 0.25 & 0.70 & $\cdot$ & $=$ & 0.45 \\
\hline LOI & 0.02 & 0.02 & 0.03 & 0.06 & 0.16 & 0.07 & 0.12 & 0.37 & 0.16 & 0.29 & 0.40 & 0.40 \\
\hline Total & 99.87 & 99.72 & 99.62 & 99.80 & 100,02 & 99.98 & 100.00 & 100.16 & 99.84 & 99.14 & 99.52 & 100.35 \\
\hline Mg \# & 46.40 & 47.24 & 40.98 & 55.35 & 14.79 & 24.43 & 21.90 & 17.69 & 46.50 & 49.37 & 51.76 & 30.38 \\
\hline $\mathrm{Cr}$ & - & - & - & - & 5 & & 5 & 5 & 80 & 48 & 41 & 18 \\
\hline $\mathrm{Ni}$ & - & - & $\cdot$ & - & 50 & - & 47 & 47 & 73 & 24 & 24 & 55 \\
\hline Co & 46 & 36 & 21 & 26 & - & 31 & $\cdot$ & - & - & - & $\cdot$ & - \\
\hline $\mathrm{v}$ & 120 & 72 & 20 & 57 & - & 10 & - & - & - & - & - & \\
\hline $\mathbf{K}$ & 15772 & 51468 & 48148 & 30715 & 35696 & 30715 & 48978 & 42337 & 19093 & - & 33205 & 9131 \\
\hline $\mathbf{R b}$ & 51 & 160 & 140 & 120 & 160 & 96 & 120 & 100 & 48 & 125 & 102 & 18 \\
\hline $\mathrm{Cs}$ & - & $=$ & - & $=$ & $=$ & 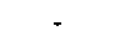 & $=$ & $=$ & - & 5.00 & 5,00 & - \\
\hline $\mathrm{Ba}$ & 896 & 1970 & 1433 & 1075 & 840 & 896 & 1400 & 1100 & 1610 & 1863 & 1263 & 160 \\
\hline Sr & 930 & 520 & 480 & 390 & 290 & 150 & 170 & 140 & 710 & 517 & 645 & 170 \\
\hline $\mathrm{Ta}$ & - & - & - & - & - & - & - & . & - & 5.00 & 5.00 & - \\
\hline $\mathrm{Nb}$ & 10 & 12 & 10 & 11 & 14 & 15 & 10 & 10 & 18 & 11 & 7 & 48 \\
\hline $\mathrm{Hf}$ & - & $=$ & - & $=$ & $=$ & - & - & $=$ & - & 8.00 & 8.00 & - \\
\hline $\mathrm{Zr}$ & 140 & 160 & 130 & 300 & 260 & 330 & 140 & 170 & 280 & 185 & 275 & 110 \\
\hline $\mathbf{T i}$ & 4916 & 2518 & 1139 & 2998 & 2158 & 2398 & 1439 & 779 & 8993 & - & 1559 & 12589 \\
\hline $\mathrm{Y}$ & 10 & 10 & 10 & 10 & 42 & 10 & 15 & Il & 25 & 11 & 16 & 13 \\
\hline Th & - & - & - & - & - & - & - & - & - & 5.00 & 72.00 & - \\
\hline $\mathbf{U}$ & - & - & - & $=$ & - & - & - & - & - & 10.00 & 10.00 & - \\
\hline $\mathrm{La}$ & 19.45 & 15.36 & 15.60 & 16.93 & - & 5.33 & $=$ & 51.46 & - & 35.14 & 126.20 & 29.06 \\
\hline $\mathrm{Ce}$ & 45.59 & 33,04 & 26.23 & 35.23 & $=$ & 12.05 & - & 100.60 & - & 75.08 & 246.50 & 71.34 \\
\hline Nd & 20.93 & 14.42 & 9.11 & 13.15 & - & 5.23 & $\cdot$ & 34.66 & . & 29.27 & 64.13 & 39.90 \\
\hline $\mathrm{Sm}$ & 3.22 & 2.48 & 1.48 & 1.92 & $=$ & 1.09 & $=$ & 3.89 & - & 4.61 & 8.53 & 7.97 \\
\hline Eu & 1.14 & 0.99 & 0.77 & 0.73 & - & 0.52 & - & 1.35 & $=$ & 1.06 & 1.12 & 1.63 \\
\hline $\mathrm{Gd}$ & 1.94 & 1.84 & 1.09 & 1.16 & - & 0.93 & - & 1.97 & - & 2.54 & 3.38 & 6.49 \\
\hline Dy & 1.03 & 1.03 & 0.79 & 0.75 & - & 0.44 & - & 0.97 & - & 1.39 & 1.71 & 5.35 \\
\hline Ho & 0.17 & 0.19 & 0.19 & 0.14 & $=$ & 0.08 & - & 0.14 & - & 0.18 & 0.27 & .96 \\
\hline Er & $0, \mathbf{4 3}$ & 0.48 & 0.63 & 0.36 & - & 0.21 & $=$ & 0.29 & - & 0.40 & 0.49 & 2.29 \\
\hline $\mathrm{Yb}$ & 0.31 & 0.42 & 0.46 & 0.23 & - & 0.17 & - & 0.26 & - & 0.20 & 0.24 & 1.65 \\
\hline $\mathrm{Lu}$ & 0.06 & 0.10 & 0.08 & 0.04 & - & 0.06 & - & 0.06 & - & 0.03 & 0.03 & 0.23 \\
\hline
\end{tabular}


Table 4- Geochemical data of basic and ultrabasic granulitic rocks. Underlined values represent the detection limit of the laboratory

Tabela 4- Dados geoquímicos dos granulitos básicos e ultrabásicos do JFC. Os valores sublinhados representam o limite de deteç̧ão do laboratório.

\begin{tabular}{|c|c|c|c|c|c|c|c|c|c|c|c|c|c|}
\hline \multirow[b]{2}{*}{ Sample } & \multicolumn{8}{|c|}{ GROUP B1 } & \multicolumn{4}{|c|}{ GROUP B2 } & \multirow[b]{2}{*}{$\begin{array}{c}\text { VAL I } \\
94\end{array}$} \\
\hline & $\begin{array}{l}\text { CPH } \\
118\end{array}$ & $\underset{94}{\text { NSAC M }}$ & $\begin{array}{c}\text { BPCM } \\
151\end{array}$ & $\begin{array}{l}\text { RPM- } \\
\text { 520-B }\end{array}$ & $\underset{534}{\text { RPM- }}$ & $\underset{\text { 503A }}{\text { VAL-M- }}$ & $\begin{array}{c}\text { VAL-M- } \\
\text { 503- }\end{array}$ & $\begin{array}{c}\text { BP-CM- } \\
\text { 166B }\end{array}$ & $\begin{array}{c}\text { CJE-44- } \\
1\end{array}$ & $\begin{array}{c}\text { CJE-44- } \\
11\end{array}$ & $\underset{204}{\text { CON- }}$ & $\begin{array}{l}\text { NSA-5- } \\
\text { 9D }\end{array}$ & \\
\hline $\mathrm{SiO}_{2}$ & 38.40 & 49.90 & 51.10 & 49.00 & 49.20 & 49.80 & 49.30 & 50.00 & 47.80 & 48.00 & 48.70 & 48.70 & 49.90 \\
\hline $\mathrm{TiO}_{2}$ & 1.80 & 0.88 & 0.59 & 1.50 & 1.40 & 1.80 & 1.80 & 1.80 & 3.50 & 3.50 & 4.50 & 3.30 & 1.20 \\
\hline $\mathrm{Al}_{2} \mathrm{O}_{3}$ & 12.20 & 15.10 & 13.20 & 15.00 & 14.60 & 13.80 & 13.40 & 14.20 & 12.70 & 12.40 & 14.30 & 12.90 & 18.30 \\
\hline $\mathrm{Fe}_{2} \mathrm{O}_{3}$ & 11.80 & 2.10 & 1.80 & 4.70 & 2.90 & 4.30 & 5.50 & 1.30 & 9.10 & 6.70 & 1.50 & 5.80 & 2.50 \\
\hline $\mathrm{FeO}$ & 8.70 & 8.00 & 8.40 & 7.80 & 9.10 & 9.80 & 9.80 & 12.50 & 7.10 & 10.00 & 10.40 & 10.10 & 9.50 \\
\hline $\mathrm{MnO}$ & 0.21 & 0.21 & 0.22 & 0.20 & 0.20 & 0.21 & 0.21 & 0.28 & 0.28 & 0.27 & 0.23 & 0.29 & 0.28 \\
\hline $\mathrm{MgO}$ & 11.40 & 8.70 & 8.40 & 6.30 & 6.80 & 5.60 & 5.50 & 5.50 & 3.90 & 3.70 & 3.90 & 4.10 & 7.00 \\
\hline $\mathrm{CaO}$ & 12.90 & 11.40 & 11.60 & 11.60 & 11.80 & 9.30 & 9.10 & 10.10 & 8.40 & 8.10 & 8.60 & 6.90 & 7.80 \\
\hline $\mathrm{Na}_{2} \mathrm{O}$ & 0.87 & 1.80 & 2.80 & 2.40 & 2.50 & 3.30 & 3.20 & 2.90 & 4.00 & 4.10 & 2.70 & 4.10 & 1.20 \\
\hline $\mathrm{K} 2 \mathrm{O}$ & 0.13 & 0.58 & 0.48 & 0.19 & 0.16 & 0.86 & 0.83 & 0.33 & 1.40 & 1.40 & 1.70 & 1.40 & 0.63 \\
\hline $\mathrm{P}_{2} \mathrm{O}_{5}$ & 0.13 & 0.06 & 0.05 & 0.11 & 0.12 & 0.24 & 0.23 & 0.23 & 0.70 & 0.73 & 2.10 & 0.58 & 0.09 \\
\hline $\mathrm{H}_{2} \mathrm{O}$ & 0.89 & 0.89 & 0.34 & 0.76 & 0.53 & 0.30 & 0.45 & 0.48 & 0.48 & 0.55 & 0.72 & 0.67 & - \\
\hline $\mathrm{CO}_{2}$ & 0.25 & 0.25 & 0.45 & 0.25 & 0.55 & 0.40 & 0.40 & 0.25 & 0.45 & 0.25 & 0.25 & 0.80 & - \\
\hline LOI & 0.06 & 0.08 & 0.10 & 0.02 & 0.02 & - & 0.05 & 0.09 & 0.35 & 0.33 & 0.16 & 0.08 & 0.44 \\
\hline Total & 99.44 & 99.95 & 99.53 & 99.83 & 99.88 & 99.71 & 99.77 & 99.96 & 100.16 & 100.03 & 99.76 & 99.72 & 98.84 \\
\hline $\mathbf{M g} \#$ & 51.26 & 61.05 & 59.90 & 48.27 & 50.86 & 42.20 & 39.92 & 41.76 & 31.25 & 29.15 & 37.17 & 32.29 & 51.50 \\
\hline $\mathrm{Cr}$ & - & 320 & 450 & & - & - & - & 210 & 37 & 33 & 11 & - & 192 \\
\hline $\mathrm{Ni}$ & $\cdot$ & 110 & 140 & & - & - & - & 71 & 61 & 56 & 49 & - & 24 \\
\hline Co & 123 & - & $\cdot$ & 72 & 72 & 77 & 77 & - & $\cdot$ & - & - & 72 & $\cdot$ \\
\hline $\mathrm{V}$ & 290 & - & - & 320 & 300 & 290 & 280 & $\therefore$ & - & - & - & 280 & - \\
\hline $\mathrm{K}$ & 1079 & 4815 & 3985 & 1577 & 1328 & 7139 & 6890 & 2739 & 11622 & 11622 & 14112 & 11622 & - \\
\hline $\mathbf{R b}$ & 5 & 13 & 12 & 9 & 8 & 19 & 19 & 12 & 22 & 20 & 37 & 20 & 28 \\
\hline $\mathrm{Cs}$ & $\cdot$ & $\cdot$ & - & $\cdot$ & $\cdot$ & - & - & - & - & - & - & - & 5.00 \\
\hline $\mathrm{Ba}$ & 45 & 20 & 100 & 72 & 72 & 493 & 493 & 20 & 370 & 390 & 570 & 412 & 196 \\
\hline $\mathrm{Sr}$ & 160 & 150 & 120 & 180 & 140 & 210 & 190 & 130 & 110 & 130 & 430 & 97 & 194 \\
\hline $\mathbf{T a}$ & - & $\cdot$ & $\cdot$ & - & $\cdot$ & $\cdot$ & - & - & $\cdot$ & - & $\cdot$ & $\cdot$ & 5.00 \\
\hline $\mathrm{Nb}$ & 11 & 10 & 10 & 10 & 10 & 10 & 14 & 10 & 43 & 33 & 25 & 93 & 20 \\
\hline Hf & - & $\cdot$ & - & - & - & - & - & - & - & - & - & - & 8.00 \\
\hline $\mathrm{Zr}$ & 68 & 37 & 30 & 83 & 79 & 170 & 160 & 100 & 230 & 300 & 140 & 270 & 129 \\
\hline $\mathrm{Ti}$ & 10791 & 5276 & 3537 & 8993 & 8393 & 10791 & 10791 & 10791 & 20983 & 20983 & 26978 & 19784 & - \\
\hline $\mathrm{Y}$ & 17 & 10 & 10 & 19 & 21 & 33 & 32 & 16 & 50 & 49 & 32 & 140 & 7 \\
\hline Th & $\cdot$ & $\cdot$ & $\cdot$ & $\cdot$ & - & - & $\cdot$ & - & $\cdot$ & - & - & - & 5.00 \\
\hline$U$ & $=$ & $=$ & - & - & - & - & $=$ & - & - & - & - & - & 12.00 \\
\hline $\mathrm{La}$ & 13.50 & - & - & 5.72 & 5.91 & 18.14 & 16.15 & - & 32.96 & 37.85 & - & 30.95 & 14.00 \\
\hline $\mathrm{Ce}$ & 34.43 & - & - & 16.91 & 16.84 & 42.05 & 40.22 & - & 87.15 & 94.80 & - & 90.69 & 28.69 \\
\hline Nd & 16.74 & - & - & 11.24 & 10.74 & 22.19 & 20.75 & - & 54.34 & 56.77 & $\cdot$ & 61.92 & 9.43 \\
\hline $\mathrm{Sm}$ & 3.57 & - & - & 3.04 & 2.86 & 5.04 & 4.66 & - & 13.68 & 12.51 & $\cdot$ & 16.65 & 1.63 \\
\hline $\mathrm{Eu}$ & 0.76 & - & - & 0.88 & 0.80 & 1.28 & 1.17 & - & 2.19 & 2.89 & - & 1.94 & 0.86 \\
\hline Gd & 3.11 & - & $\cdot$ & 3.08 & 2.87 & 4.65 & 4.22 & - & 13.87 & 11.32 & - & 16.96 & 1.17 \\
\hline Dy & 2.70 & - & - & 3.17 & 2.86 & 4.96 & 4.29 & - & 13.74 & 12.61 & - & 22.97 & 1.04 \\
\hline Ho & 0.60 & - & - & 0.64 & 0.60 & 1.04 & 0.93 & - & 2.79 & 2.64 & - & 4.50 & 0.23 \\
\hline Er & 1.91 & $\cdot$ & - & 1.77 & 1.76 & 3.04 & 2.84 & - & 6.47 & 7.14 & - & 11.62 & 0.71 \\
\hline $\mathrm{Yb}$ & 1.51 & - & - & 1.38 & 1.39 & 2.63 & 2.50 & - & 7.10 & 6.72 & $\cdot$ & 8.86 & 0.68 \\
\hline Lu & 0.20 & - & - & 0.19 & 0.20 & 0.33 & 0.34 & - & 1.00 & 0.96 & - & 0.98 & 0.10 \\
\hline
\end{tabular}

triangles) are less differentiated, enriched in $\mathrm{MgO}$ and $\mathrm{MnO}$, with higher $\mathrm{Mg}$ \# (Table 4). The two samples also display relative enrichment and/or remobilization of some LILE, such as $\mathrm{K}_{2} \mathrm{O}, \mathrm{Na}_{2} \mathrm{O}$ and $\mathrm{Ba}$. The other samples of the $\mathrm{Bl}$ tholeiitic basic rocks (triangles) are progressively enriched in incompatible elements (Fig. 12a, b), probably indicating higher degree of differentiation and/or crustal contamination The chondrite normalized REE abundances of Bl group are 

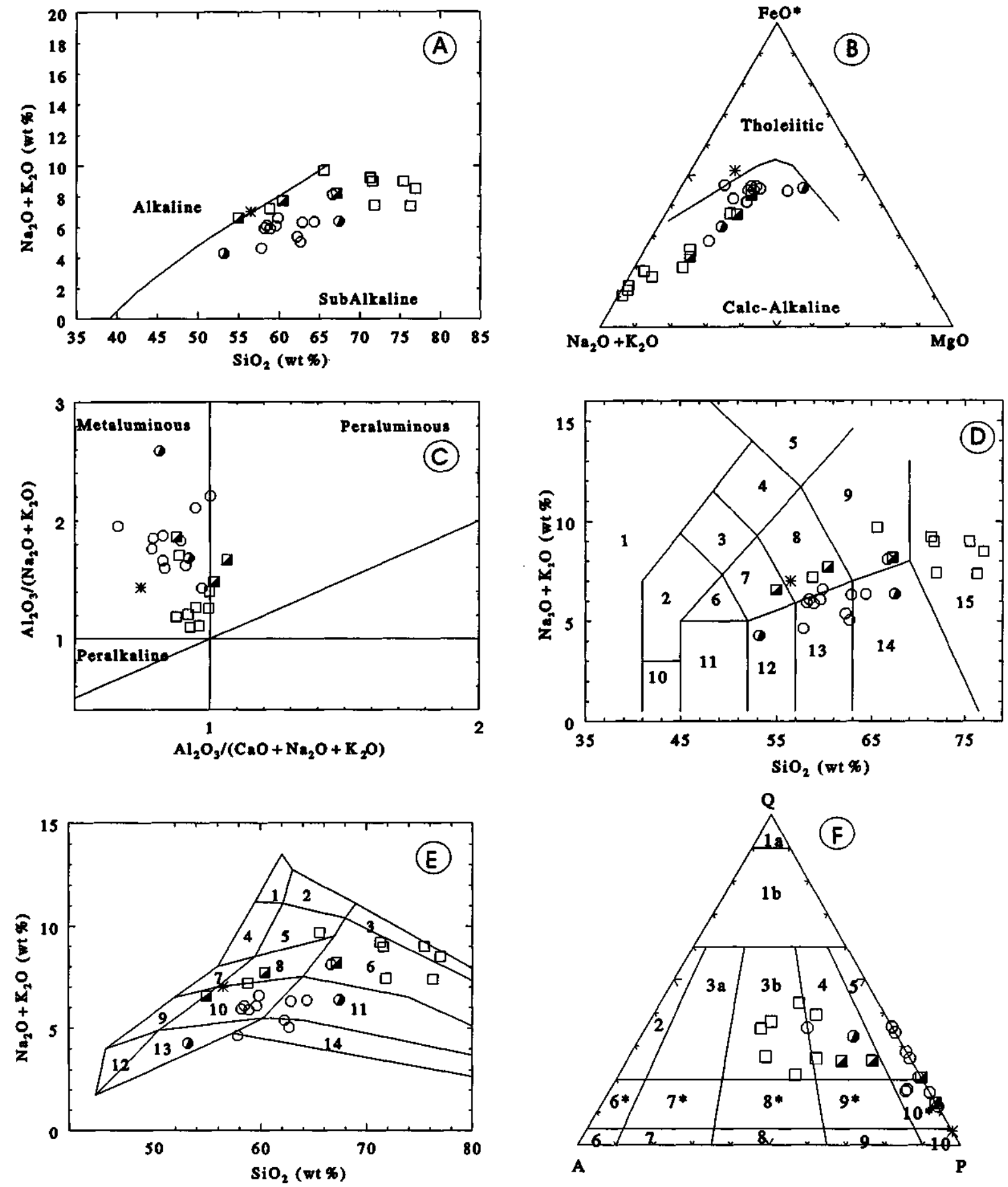

Figure 3 - (a) - Plot of acid to intermediate granulites in the Alk x SiO 2 diagram of Irvine \& Baragar (1971). (b) - AFM diagram (Irvine \& Baragar 1971) with the distribution of the calc-alkaline series, (c) - Metaluminous character of the two calc-alkaline series (Maniar \& Piccoli 1989). (d) - Plot of the calc-alkaline series within the Alk $x \mathrm{SiO}_{2}$ chemical classification diagram (Le Maitre 1989), Legend: 1 -foidite; 2 - tephrite and basanite, 3 - phono-tephrite, 4 - tephri-phonolite, 5 - phonolite, 6 - trachy-basalt, 7 - basaltic trachy-andesite, 8 - trachy-andesite, 9 - trachyte and trachy-dacite 10 - picrobasalt, 11 - basalt, 12 - basaltic andesite, 13 - andesite, 14 - dacite, 15 - rhyolite. (e) -Plot of the calc-alkaline series within the Alk $x \mathrm{SiO}_{2}$ chemical classification diagram (Middlemost 1985). Legend: 1 - alkali feldspar syenite, 2 - alkali feldspar quartz syenite, 3 - alkali feldspar granite, 4 - syenite, 5 - quartz syenite, 6 - granite, 7 - monzonite, 8 - quartz monzonite, 9 - monzodiorite, 10 - quartz monzodiorite, 11 - granodiorite, 12 - diorite andgabbro, 13 - quartz diorite, 14 - tonalite. (f) - QAP normative diagram for rocks of the calc-alkaline series. Symbols: CAS1 - circles and half-filled circles, CSA2 - squares and half-filled squares; Leucossome associated to B2 basic rocks - asterisk. Figura 3 - (a) - Granulitos félsicos a intermedianas no diagrama Alk x SiO${ }_{2}$ de Irvine \& Baragar (1971). (b) - Diagrama AFM (Irvine \& Baragar 1971) com a distribuição das séries cálcio-alcalinas. (c) - Caráter metaluminoso das duas séries cálcio-alcalinas no diagrama de Maniar \& Piccoli (1989). (d) - As séries

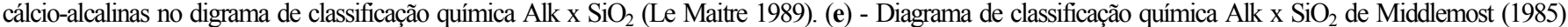
para as rochas granulíticas intermediárias a ácidas. (f) - Diagrama QAP normative para as rochas das séries cálcio-alcalinas. Símbolos: CAS1 -círculos e círculos parcialmente preenchidos; CAS2 -quadrados e quadrados parcialmente preenchidos; Leucossoma associado às rochas básicas do grupo B2 - asterisco. 


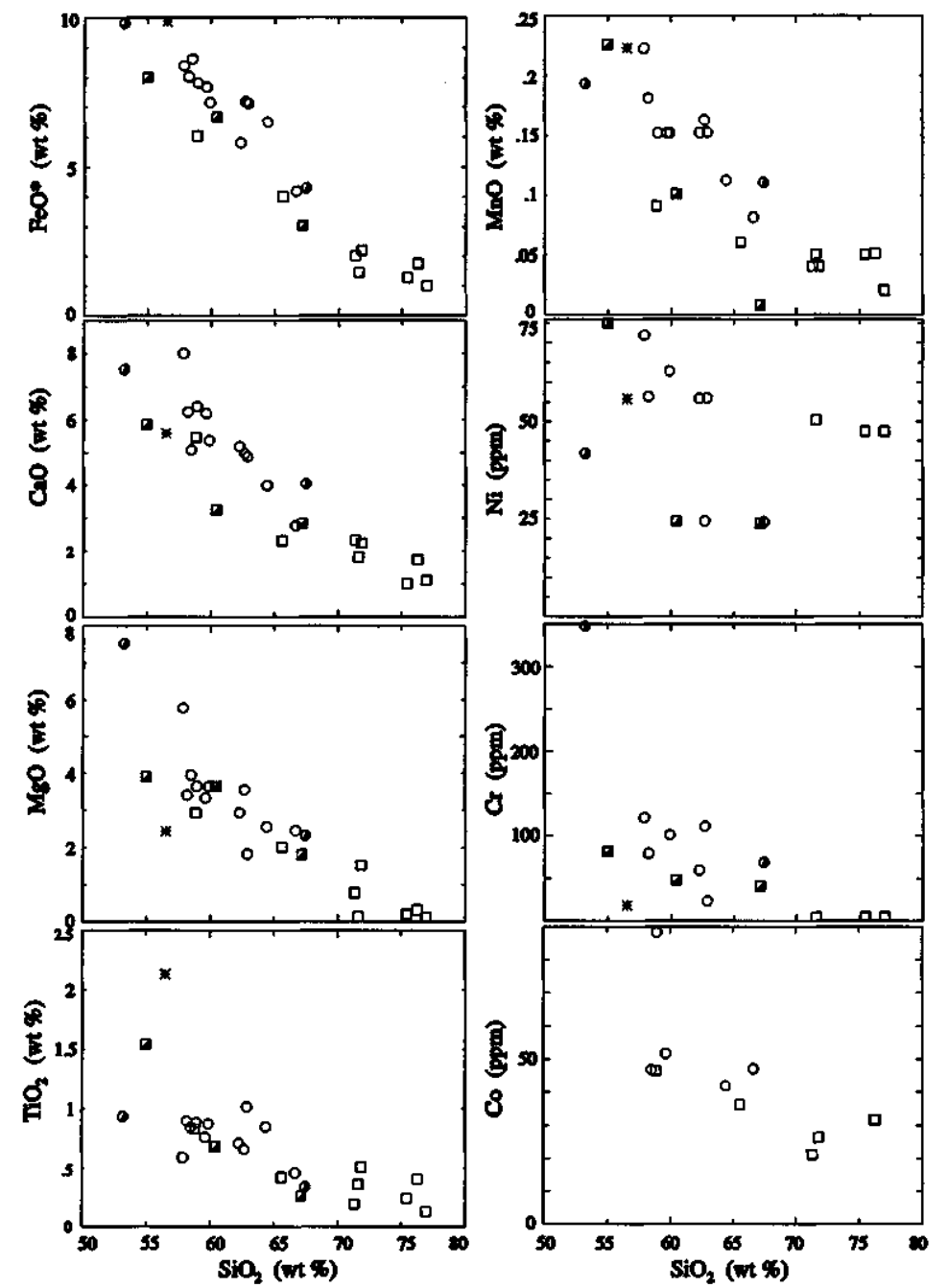

(A) D

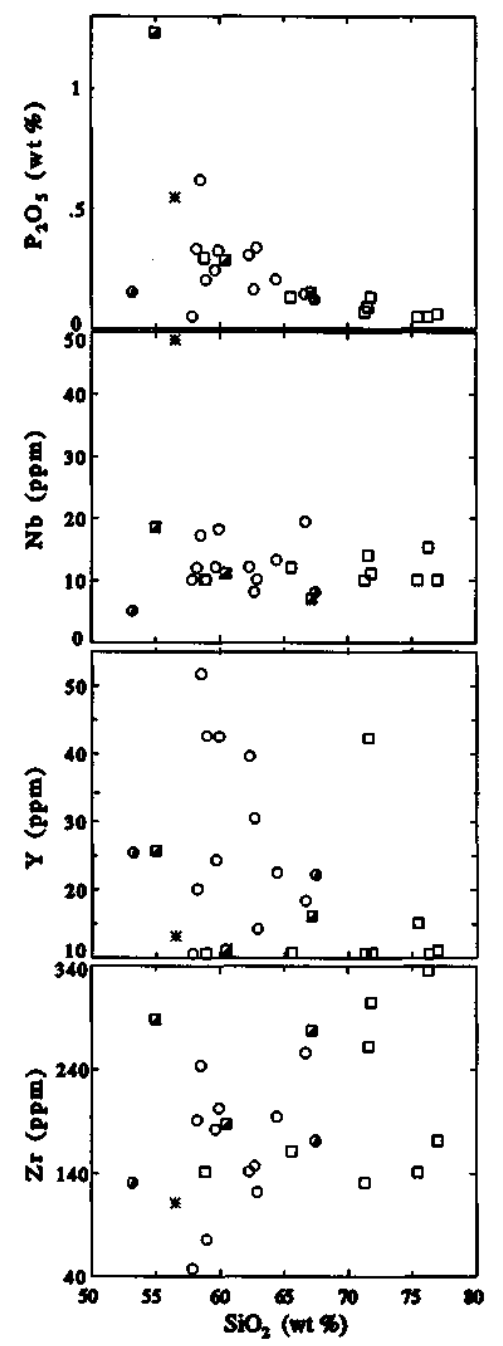

(B)
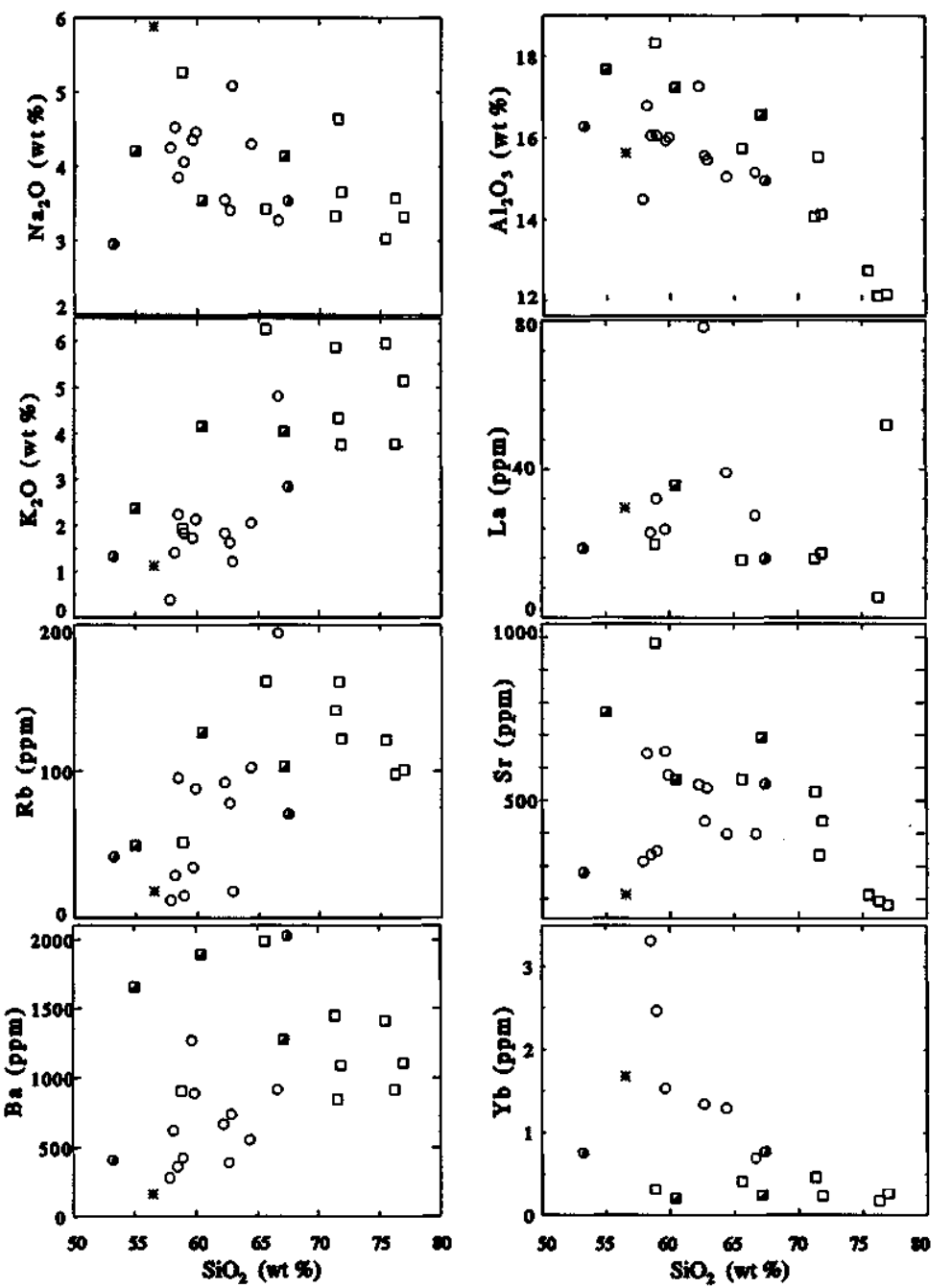

\& 四 今 

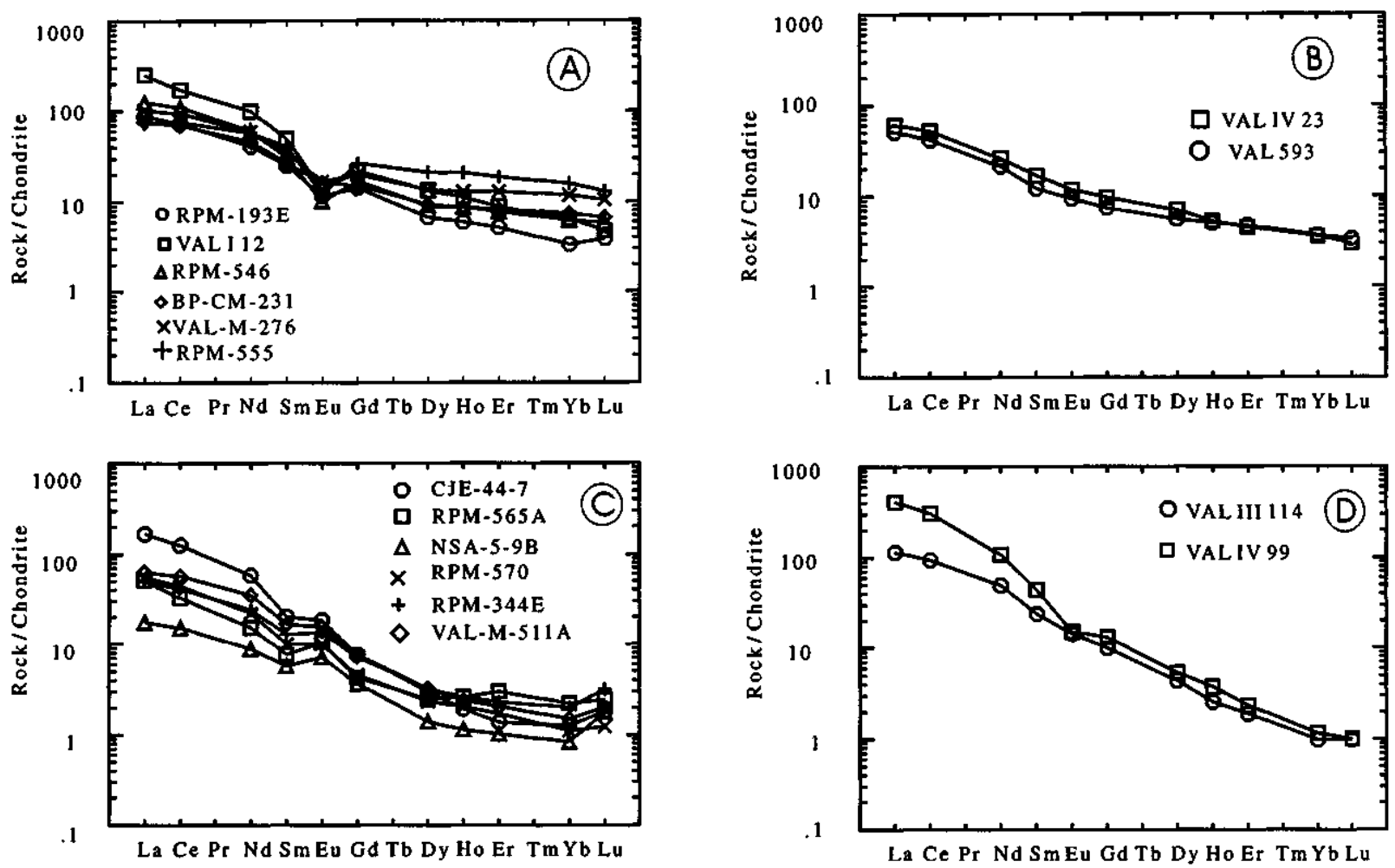

Figure 5 - Chondrite-normalized (Boynton 1984) REE signature of the -alkaline series: (a) CAS1; (b) anomalous samples of CAS1, (c) CAS2, and (d) anomalous samples of CAS2

Figura 5 - Padrões de abundância dos REE normalizados por condrito (Boynton 1984) das séries calcio-alcalinas: (a) CASl, (b) amostras anômalas das CASl, (c) CAS2, e (d) amostras anómalas da CAS2.

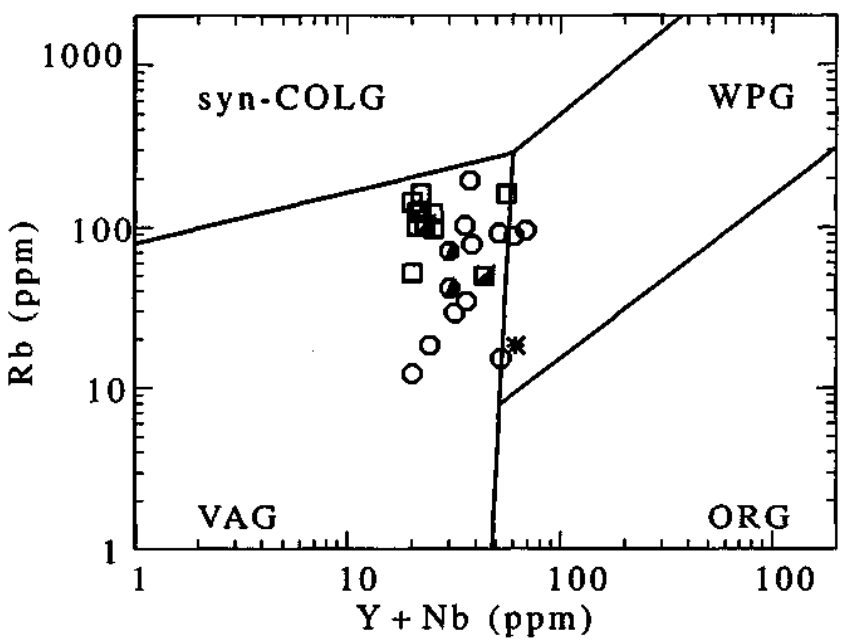

Figure 6 - Plot of the calc-alkaline series within the Rb $x Y+$ $\mathrm{Nb}$ tectonic discriminant diagram (Pearce et al. 1984). Legend: sin-col- sin-collisional granitoids, WPG- within plate granitoids, VAG- volcanic arc granitoids, ORG- ocean ridge granitoids. Symbols as in figure 3.

Figura 6 - Rochas calcio-alcalinas plotadas no diagrama discriminante $\mathrm{Rb} \times$ $\mathrm{Y}+\mathrm{Nb}$ (Pearce et al. 1984). Símbolos como na figura 3.

highly fractionated, and as compared to B2 group, more depleted in total REE and have a smaller negative Eu anomalies $\left(\mathrm{Eu} / \mathrm{Eu}^{*} \approx 0.81-0.88\right)$ (Fig. 13).

One single ultramafic sample (filled inverted triangle) is composed only (Table 1) by clinopyroxene, green amphibole and ilmenite. In the Jensen's diagram, it classifies as a komatiitic basalt (Fig. 11), while in the le Maitre's diagram it plots

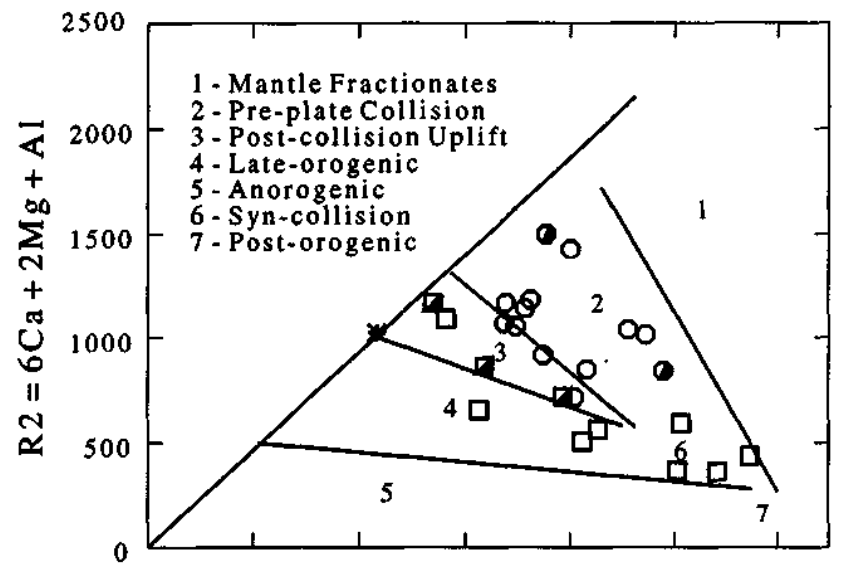

$\begin{array}{lllllll}0 & 500 & 1000 & 1500 & 2000 & 2500 & 3000\end{array}$ $\mathrm{R} 1=4 \mathrm{Si}-11(\mathrm{Na}+\mathrm{K})-2(\mathrm{Fe}+\mathrm{Ti})$

Figure 7-R1 x R2 diagram (Batchelor \& Bowden 1985) with the tectonic discrimination of the calc-alkaline series Symbols as in figure 3.

Figura 7 - Ambientação tectônica das séries calcio-alcalinas segundo o diagrama discriminante R1 x R2 de Batchelor \& Bowden (1985). Símbolos como na figura 3 .

in the meimechite and komatiitic fields. However the lower content of $\mathrm{MgO}$ ( $<$ than komatiitic rocks), the higher contents of $\mathrm{Fe}_{2} \mathrm{O}_{3}, \mathrm{FeO}, \mathrm{TiO}_{2}, \mathrm{CaO}, \mathrm{V}$, and its mineralogy suggest cumulatic processes derived from rocks of the $\mathrm{B} 1$ group.

The $\mathbf{B 2}$ group (asterisks) comprises alkaline to transitional high $\mathrm{TiO}_{2}-\mathrm{P}_{2} \mathrm{O}_{5}$ basic rocks enriched in HFS elements (Figs. $9,12 \mathrm{a}$ and $12 \mathrm{~b}$ ), including sieno and monzo-gabbros (R1R2 


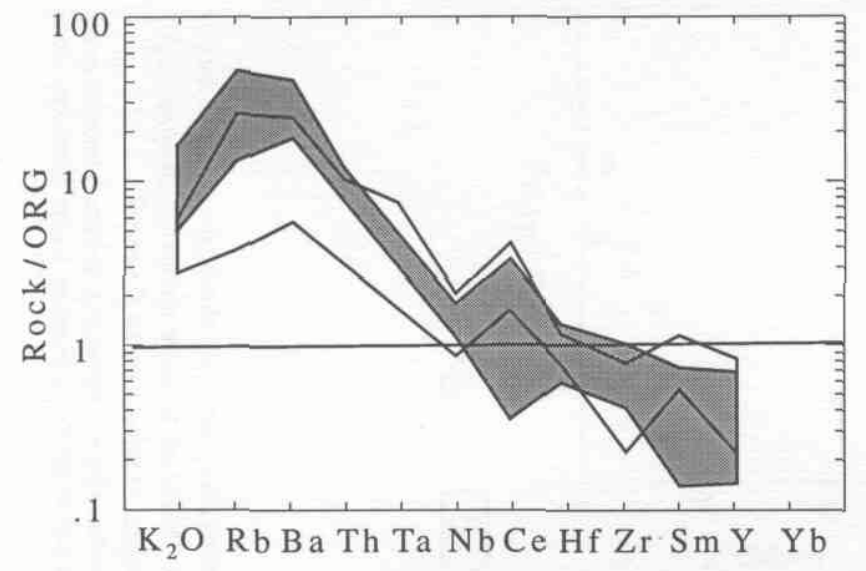

Figure 8 - Ocean Ridge Granite (Pearce etal. 1984) normalized multielemental spidergrams of the calc-alkaline series. The CAS1 and CAS2 (gray shaded area) exhibit patterns similar to those of volcanic arc granitoids.

Figura 8 - Aranhogramas normalizados por Ocean Ridge Granite (pearce et al. 1984) para as duas sáries calcio-alcalinas CAS1 e CAS2 (área cinza), que apresentam padrões semelhantes aos granitóides de arcos vulcânicos.

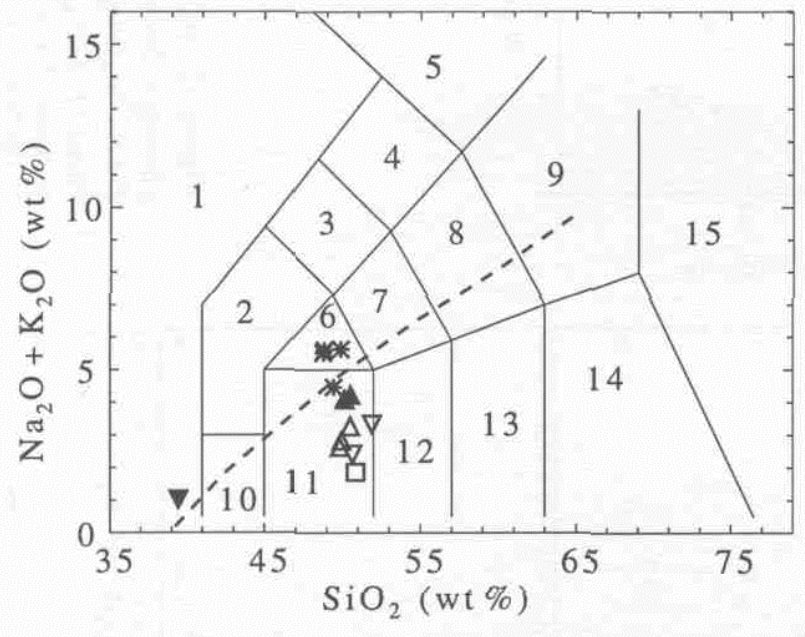

Figure 9 - Classification of basic rocks in the Alk $x \mathrm{SiO}_{2}$ diagram of Le Maitre (1989). The dashed line is the boundary between alkaline and sub-alkaline fields (Irvine \& Baragar 1971). Symbols: B1 group -triangles; B2 group - asteriscs, High alumina basalt -open, square, cumulatic rock - inverted filled triangle. Fields:1 - Foidite, 2 - Tephrite and Basanite 3 -Phonotephrite, 4 -Tephriphonolite, 5 - Phonolite, 6 Trachybasalt, 7 -Basaltic trachyandesite, 8 -Trachyandesite, 9 - Trachyte, 9 - Picrite, 11 - Basalt, 12 -Basaltic Andesite, 13 -Andesite, 14 - Dacite and 15 -Rhyolite.

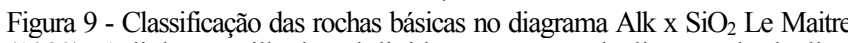
(1989). A linha pontilhada subdivide os campos alcalino e sub-alcalino segundo Irvine \& Baragar (1971). Símbolos grupo Bl- triângulos, grupo $\mathrm{B} 2$-asteriscos, basalto rico em alumina- quadrado, cumulado ultrabásicotriângulo preto invertido.

diagram of De La Roche et al. 1980, not shown). Figures 12a and $12 \mathrm{~b}$ indicate that, in comparison with the $\mathrm{Bl}$ group, the $\mathrm{B} 2$ rocks are high $\mathrm{TiO}_{2}(>3.3 \%), \mathrm{K}_{2} \mathrm{O}(1.1-1.7 \%), \mathrm{FeO}^{*}$ $(>10 \%), \mathrm{P}_{2} \mathrm{O}_{5}(>0.58 \%)$, and enrichmed in incompatible elements, such as $\mathrm{Rb}, \mathrm{Zr}, \mathrm{Ba}, \mathrm{Nb}, \mathrm{Y}$ and REE. Normalized to chondrite, their REE abundance (Fig. 13) displays bulk enrichment, less fractionated patterns, flatter HREE distribution, and negative Eu anomalies $\left(\mathrm{Eu} / \mathrm{Eu}^{*} \approx 0.74\right.$ and 0.31$)$.

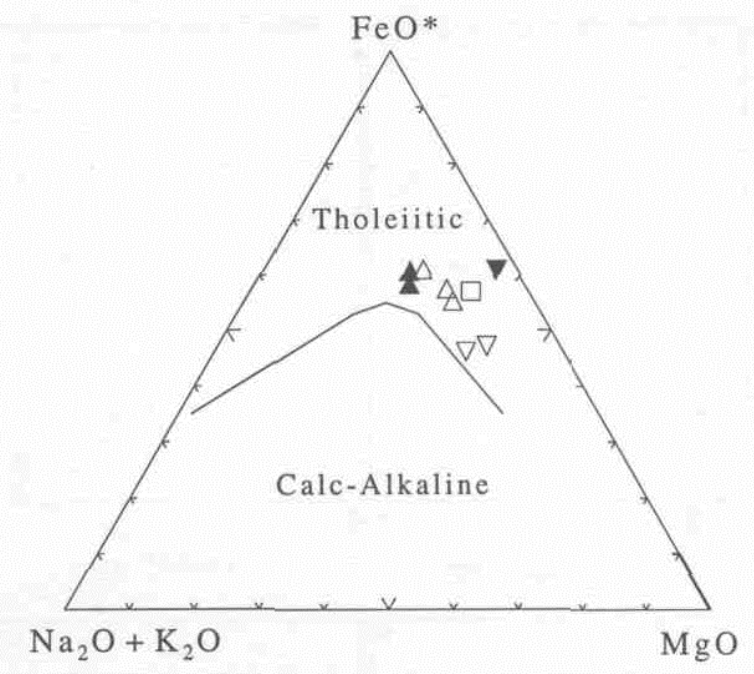

Figure 10- Plot of the basic sub-alkaline granulitic rocks in the AFM diagram. Symbols as in figure 9.

Figure 10- Diagrama AFM de Irvine \& Baragar (1971) com as amostras dos granulitos básicos.sub-alcalinos Símbolos como na figura 9.

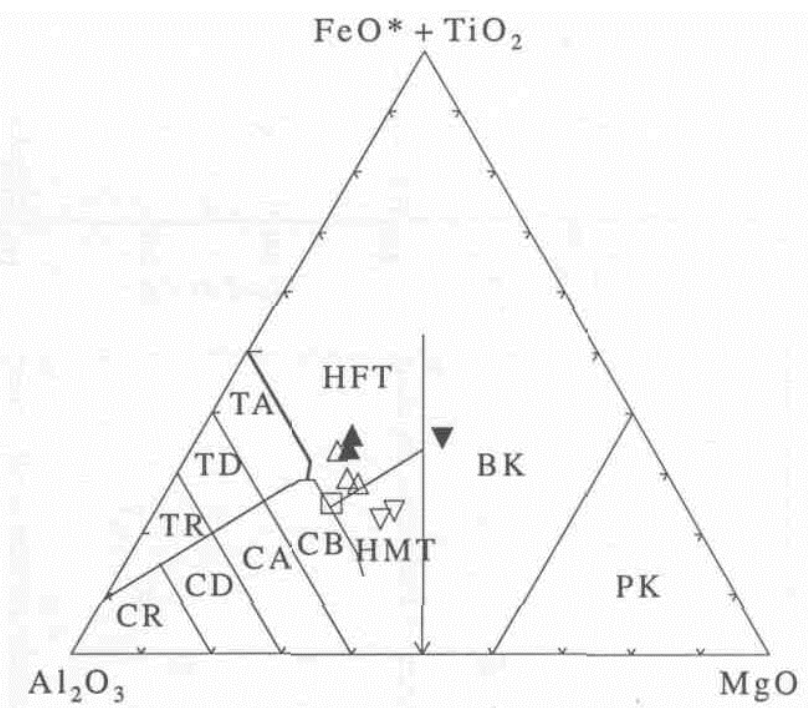

Figure 11- The cationic diagram of Jensen (1976) separates some samples of B1 group that plot within the HMT field (High MgO tholeiites) and one sample that plots in the calcalkaline field. Symbols as in figure 9.

Figure 11- O diagrama catiônico de Jensen (1976) separa algumas rochas do grupo B1 que plotam no campo dos HMT e uma amostra que plota no campo calcio-alcalino. Simbolos como na figura 9.

One sample (Val-I-594) of a high alumina calc-alkaline gabbroic granulite (open square), has a chemical composition (Figs. 12a and 12b) and chondrite-normalized REE abundance (Fig. 13) more consistent with the CAS2 series. This association is stronger if the diagrams of figures $14 \mathrm{a}$ to $14 \mathrm{~d}$ are considered.

In spite of uncertainties about the occurrence of primary liquids or differentiates and modifications due to two metamorphic pulses, the basic rocks of the Juiz de Fora complex were plotted in tectonic discriminant diagrams (Fig. 14). The $\mathrm{Bl}$ samples disperse in the intraplate, ocean floor and island arc fields. The more LILE-enriched samples (triangles) plot in the E-MORB and intraplate fields, while the most LILEdepleted samples plot also in the island arc basalt field. 

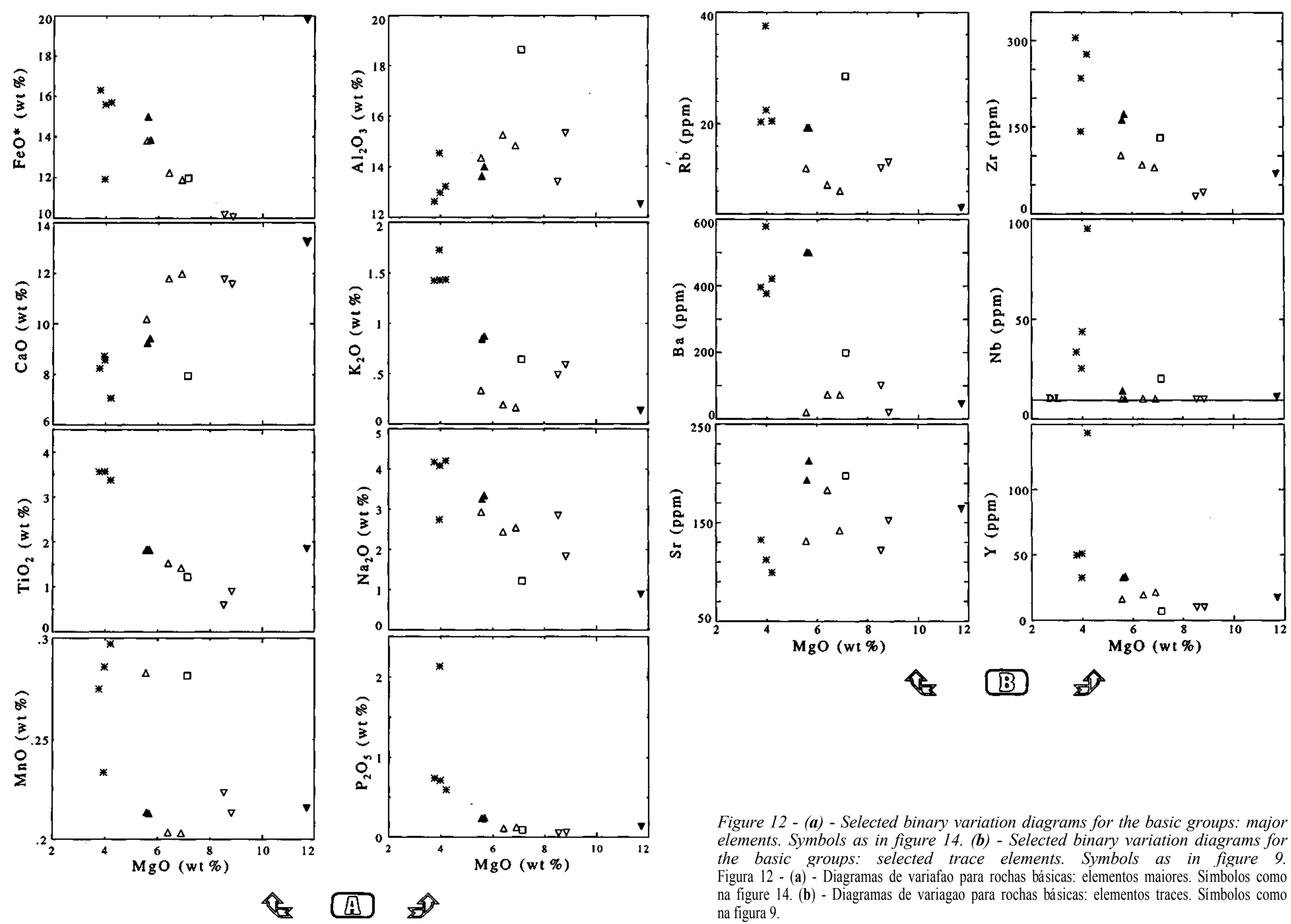
elements. Symbols as in figure 14. (b) - Selected binary variation diagrams for the basic groups: selected trace elements. Symbols as in figure Figura 12 - (a) - Diagramas de variafao para rochas básicas: elementos maiores. Símbolos como na figura 9. 

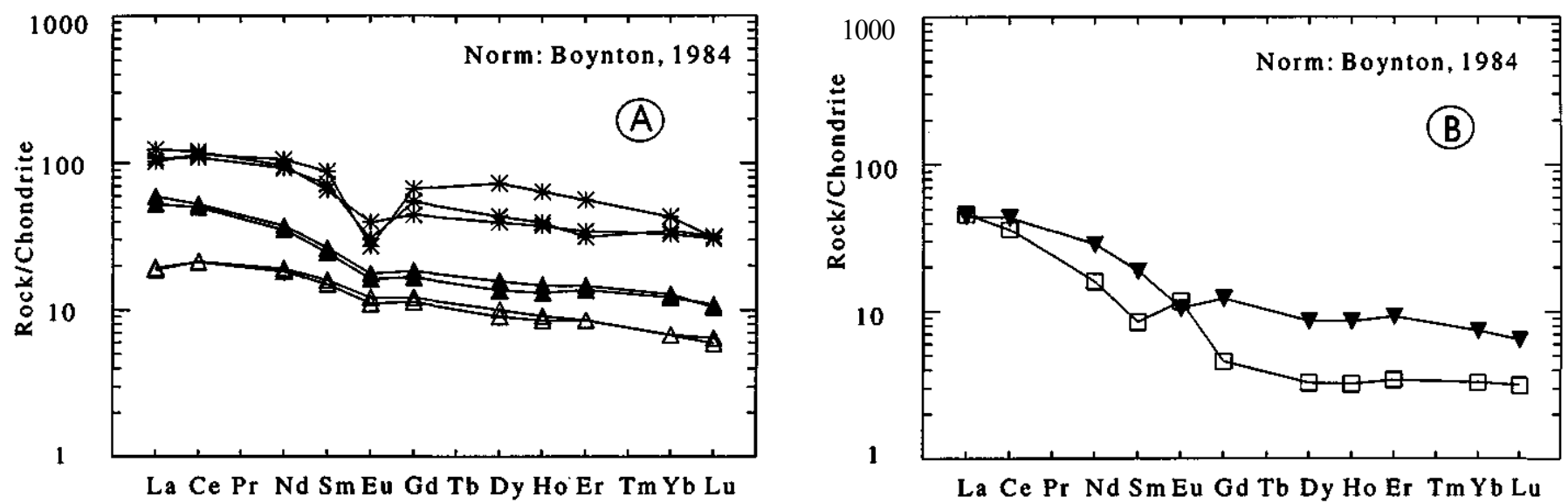

Figure 13-Chondrite -normalized (Boynton 1984) REE signature of the JFC basic rocks: a) B1 and B2 groups; b) cumulatic rock and calc-alkaline sample. Symbols as in figure 9.

Figura 13- Assinatura dos REE normalizados por condrito (Boynton 1984) para as rochas básicas do JFC: a) Grupos B1 e B2; b) Rocha cumulática e amostra calcio-alcalina. Símbolos como na figura 9.
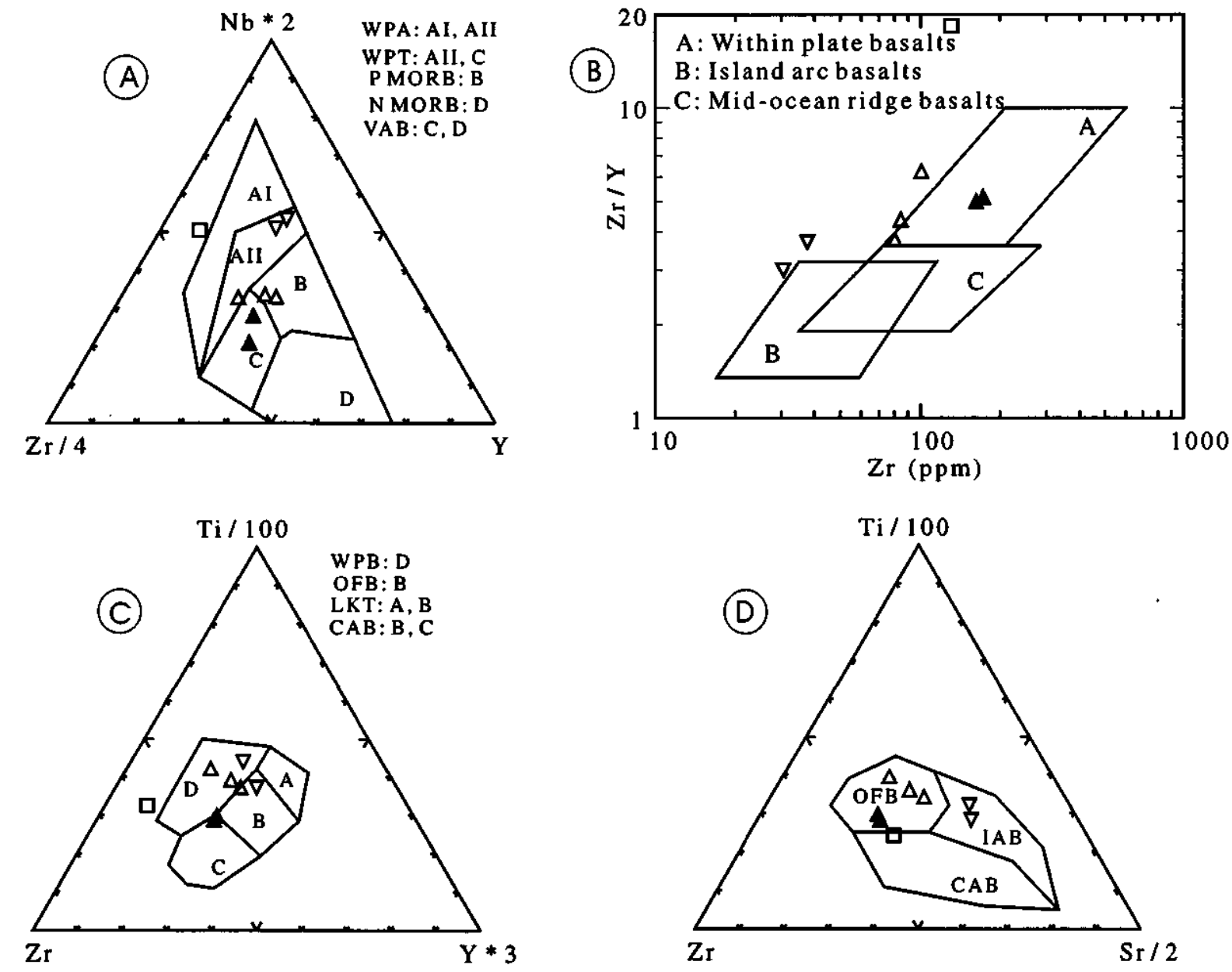

Figure 14 - (a) - Plot oftholeiitic B1 group in the 2Nb x Zr/4 x Y tectonic discriminant diagram of Meschede (1986). (b) - Zr/Yx Zr tectonic discriminant diagram (Pearce \& Norry 1979) for the basic granulitic rocks, (c) - Ti/100 x Zr x Y tectonic diagram of Pearce \& Cann (1973) for basic granulitic rocks of B1 group. Fields: OFB-Ocean Floor Basalts, WPB-Within Plate Basalts, LKT-Low-K Tholeiites, CAB-Calc-alkaline Basalts, (d) -Plot of basic groups of the B1 group in the Ti/100 $x$ ZrxSr/2 tectonic discriminant diagram of Pearce \& Cann (1973).Fields: OFB-Ocean Floor Basalts, IAB-Island Arc Basalts, CAB-Calc-alkaline Basalts. Symbols as in figure 9.

Figura 14 - (a) - Rochas básicas toléticas do grupo Bl plotadas no diagrama discriminante $2 \mathrm{Nb} \times \mathrm{Zr} / 4 \times \mathrm{Y}$ de Meschede (1986). (b) - Rochas básicas toleíticas do grupo B1 plotadas no diagrama discriminante Zr/Y x Zr de (Pearce \& Norry 1979). (c) - Rochas básicas do grupo B1 plotadas no diagrama discriminante Ti/100 x Zr x Y de Pearce \& Cann (1973). (d) - Rochas basicas do grupo Bl plotadas no diagrama discriminante Ti/100 x Zr x Sr/2 de Pearce \& Cann (1973). Símbolos como na figura 9 . 
However, as the $\mathrm{Bl}$ samples occur in continuous outcrops along the base of the uppermost granulitic thrust sheet (Fig. 2), except sample 94, they can be interpreted as formed under a common tectonic setting. The multielemental N-MORB normalized diagrams (Pearce 1983) indicate that Bl samples fit equally to E-MORB, Back-arc Basalts and Within plate tholeiites (Fig. 15a). The lack of field evidences for typical ophiolite petrotectonic associations, such as ultrabasic rocks and pelitic-chert deep marine sediments, besides tholeiitic basic rocks, suggests continental intraplate environment. The B1 rocks are also compared to Phanerozoic and Proterozoic examples of continental tholeiitic intraplate magmas (Figs. 15 band $15 \mathrm{c}$ ).

Besides the alkaline character of the $\mathrm{B} 2$ group rocks, indicated by the Alk x $\mathrm{SiO}_{2}$ ratios (Fig. 11), other diagrams that use $\mathrm{P}_{2} \mathrm{O}_{5}, \mathrm{TiO}_{2}, \mathrm{Zr}, \mathrm{La}$ and $\mathrm{Nb}$ contents (Winchester \& Floyd 1976, Rollinson 1993, Mullen 1983, not shown), show that these rocks are enriched in HFSE and LREE, and point to intraplate settings (continental alkaline or alkaline ocean is- land). The alkaline continental within-plate environment is also indicated by the N-MORB normalized expanded spidergrams (Pearce 1983), compared with modern examples of Ocean Island Basalts and Alkaline Continental Basalts (Fig. $15 d)$.

FINAL REMARKS The geochemical data of the JFC in the investigated transect reveal a complex geological evolution prior to the Brasiliano orogeny. The granulites rocks may be grouped in two tectonic associations. The calc-alkaline nature of CAS1 and CAS2 indicate a convergent tectonic setting similar to modern active margins, and the tholeiitic character of $\mathrm{B} 1$ group and alkaline of $\mathrm{B} 2$ group suggest intracontinental extensional settings.

The relationship between these two contrasting tectonic associations was obliterated by the intense shortening during the Brasiliano orogeny. However, the CAS1 has the most widespread areal distribution (Fig. 2), while the basic B1 and B2 groups concentrate respectively at the base of the upper-
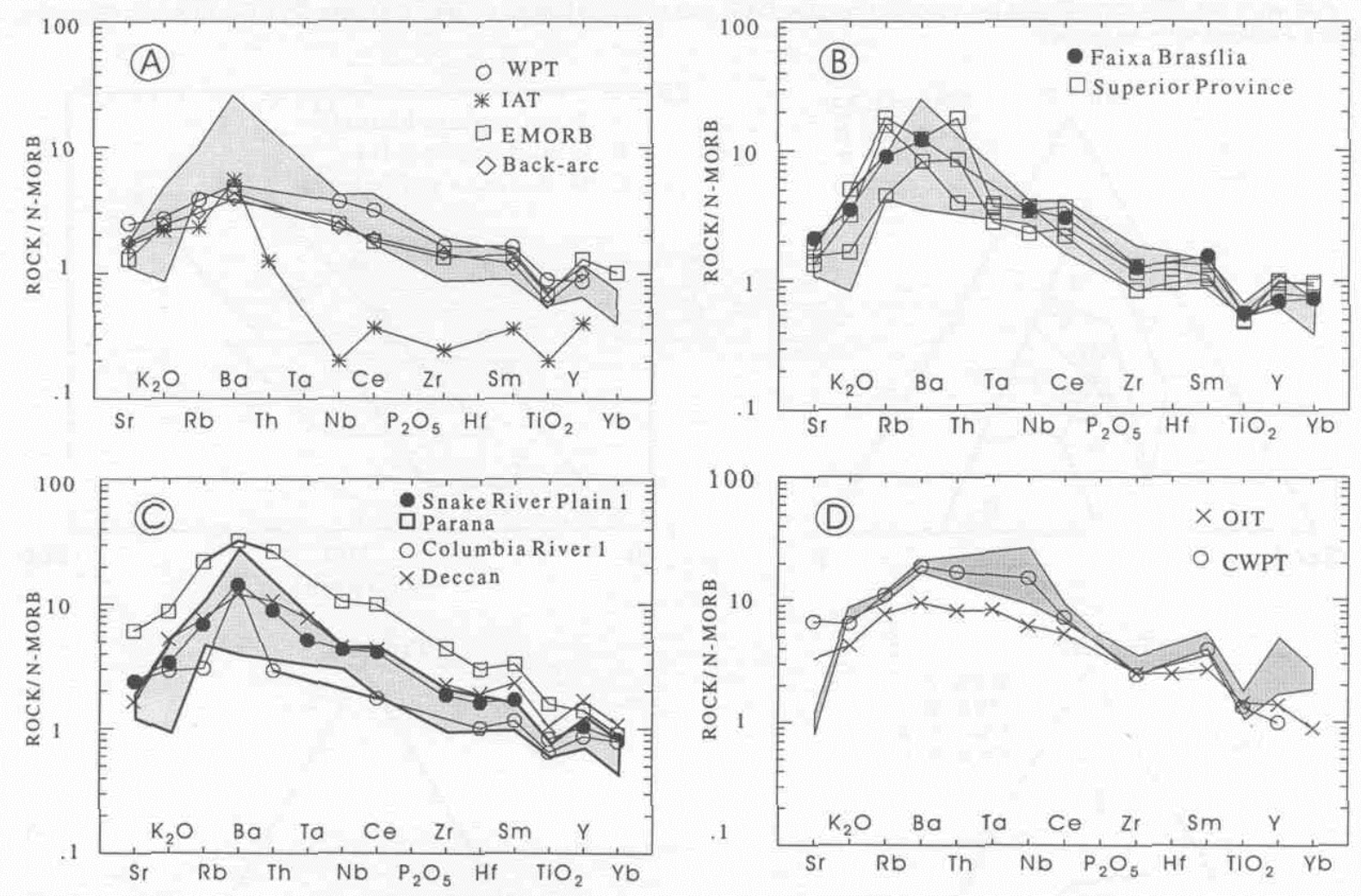

Figure 15 - (a) - N-MORB normalized (Pearce 1983) spidergrams of the B1 group (grey shaded area) compared with typical compositions of Island-arc basalt, E-MORB, Back-arc basalt and Within plate Tholeiitic basalt (data from Wilson 1988, table 6.6, p 176). (b) -N-MORB normalized (Pearce 1983) of the B1 group (grey shaded area) compared with typical compositions of Phanerozoic Continental Intaplate Basalts (Data from Wilson 1988, tables 10.2, 10.3 and 10.4, pp 299-301). (c) -N-MORB normalized (Pearce 1983) spidergrams of the B1 group (grey shaded area) compared with typical compositions of Proterozoic Continental Intraplate Basalts (data from Valeriano 1992-Low TiO fractionated Meso/Neoproterozoic basic rocks from the Nappe Passos, Brasilia belt, Brazil; and Condie et al. 1987- Proterozoic dyke swarms of Canadian shield), (d) -N-MORB normalized (Pearce 1983) spidergrams of the B2 group (grey shaded area) compared with typical compositions of Ocean Island alkaline basalt and Continental within plate alkaline basalt (Data from Wilson 1988, table 6.6, p 176).

Figura 15 - (a) - Aranhogramas multielementais normalizados por N-MORB (Pearce 1983) das rochas do grupo Bl (área cinza do diagrama), comparadas a composites típicas de basaltos de arco de ilhas, basaltos do tipo E-MORB, basaltos toleíticos intraplaca e basaltos de retro-arco. (dados extraidos da Tabela 6.6 de Wilson. 1988). (b) - Aranhogramas multielementais normalizados por N-MORB (Pearce 1983) das rochas do grupo Bl (área cinza do diagrama), comparadas a composições típicas de basaltos toleíticos continentais fanerozóicos (dados extraidos das tabelas 10.2, 10.3 e 10.4 de Wilson 1988, pp 229-301). (c) Aranhogramas multielementais normalizados por N-MORB (Pearce 1983) das rochas do grupo Bl (área cinza do diagrama), comparadas à composições típicas de basaltos toleíticos continentais proterozóicos (dados extraidos de: Valeriano 1992- Metabasitos de $\mathrm{Baixo}^{\mathrm{TiO}} \mathrm{O}_{2}$, fracionados, Meso/Neoproterozóicos da região da Nappe de Passos, Faixa Brasília; e Condie et al. 1987- enxames de diques proterozóicos do escudo canadense). (d) - Aranhogramas multielementais normalizados por N-MORB (Pearce 1983) das rochas do grupo B2 (área cinza do diagrama), comparadas a composições típicas de basaltos alcalinos de ilhas oceânicas e de ambientes intracontinentais. (dados extraidos da Tabela 6.6 de Wilson. 1988. 
most thrust sheet and at the intermediate thrusts sheet. This spatial distribution, the relict magmatic textures in CAS1 samples and its field relationship with CAS2, suggest that CAS1 represents the basement to the other calc-alkaline and basic rock assemblages, and probably the oldest segment of continental crust. It is also suggested that B1 (tholeiitic) and B2 (alkaline) sample groups may represent extensional tectonic episodes (continental flood magmatism and dykes) that took place in the older continental crust. All rocks belonging to the different tectonic environments were metamorphosed under granulite facies during the Transamazonian.

Available geochronological data for the JFC in the investigated region are one $\mathrm{U} / \mathrm{Pb}$ discordia of CAS2 with Paleoproterozoic and Neoproterozoic intercepts (Machado et al. 1996) and two $\mathrm{Rb} / \mathrm{Sr}$ errorchrons (Heilbron 1993) that corroborate the Paleoproterozoic age of the granulite facies metamorphism, overprinted on juvenile protoliths. Only detailed geochronological data associated with isotopic studies could contribute to solve the temporal succession and the sources of the other JFC rocks.
The geochemical data of studied area show that the common LILE depletion of other granulite terranes was not detected in the JFC. Except for a few CAS1 samples that have selective $\mathrm{Rb}$ removal and consequently higher $\mathrm{K} / \mathrm{Rb}$ ratios, the other granulite samples have LELE contents similar to the upper crust average (Taylor \& McLennan 1985). The Eu/Eu* ratios and the bulk composition of the studied granulites do not corroborate the lower crust residue models proposed previously. If these assumptions are correct, the Juiz de Fora Complex could be regarded as the root of a Paleoproterozoic active margin (Teixeira \& Figueiredo 1991), tectonically overthrusted during the Brasiliano orogeny.

Acknowledgments To CNPq, PADCT-FINEP, FAPESP and FAPERJ for financial support of the geological research in the Rio de Janeiro State, and to J. C. Almeida, C. Valeriano, M. Tupinamba, C. Valladares, and B. Duarte (DGRG/FGEL-UERJ) for the field work dedication during the last ten years of research. The authors also appreciate the improving suggestions of $\mathrm{C}$. Valeriano and of two anonymous reviewers.

\section{REFERENCES}

ANDREIS, R. R.; RIBEIRO, A. \& PACIULLO, F. V. P. 1989. Ciclos deposicionais no Proterozóico das folhas Barbacena e Divinópolis (setor sul), 1:250000. In: Simp. Geol. Sudeste, I, Rio de Janeiro, 1989. Boletim de Resumos... Rio de Janeiro, SBG. p. 97-98.

BARBOSA, A. L. \& SAD, J. H. G. 1983a. Petrografia dos charnockitos e rochas afins ao longo da divisa RJ/MG. IirSimp. Geol. MG., 2, Belo Horizonte, 1983. Anais... Belo Horizonte SBG. p. 63-74.

BARBOSA, A. L. \& SAD, J. H. G. 1983b. Geoquímica e petrologia dos charnockitos e rochas afins do Complexo Juiz de Fora.RJ/MG. In: Simp. Geol. Mg., 2, Belo Horizonte, 1983Anais... Belo Horizonte SBG. p. 75-84

BATCHELOR, R. A. \& BOWDEN, P. 1985. Petrogenetic interpretation of granitoid rock series using multicationic parameters. Chem, Geol., 48 : 43-55.

BOYNTON, W. V. 1984. Geochemistry of the Rare Earth Elements: meteorites studies. In: Henderson P. (ed.), Rare Earth Element Geochemistry. Elsevier, pp. 63-114.

BROWN, G. C. 1982. Calc-alkaline intrusive rocks: their diversity, evolution and relation to volcanic arcs. In: Thorpe (ed), Andesites. John Wiley \& Sons, pp: 437-461.

CAMPOS NETO, M. C. 1992. A porção ocidental da Faixa Alto Rio GrandeEnsaio de Evolução Tectônica. Tese de Doutoramento, IG-USP. 210p (inédito).

CAMPOS NETO, M. C. \& FIGUEIREDO, M. C. H. 1990. Evolução geológica dos terrenos Costeiro, Paraíba do Sul e Juiz de Fora.In:Cong. Bras. Geol, XXXVI, Natal, 1990, Ana is... Natal, RN, SBG. V.6:2631-2648.

CONDffi, C.K.; DOBROW, D.J.\& CARD, K.D. 1987. Geochemistry of Precambrian basic dykes from the southern Superior Province of the Canadian Shield. In: Halls, H.C. \& Fahrig, W.F. (eds.) Basic dyke swarms. Geol. Assoc. Canada Special Paper 34:95-108

CORDANI, U. G.; DELHAL, L. \& LEDENT, O. 1973. Orogeneses Superposhes dans le Precambrien du Brésil Sud-Oriental (Etats de Rio de Janeiro et de Minas Gerais). Rev. Bras. Geoc., 3(1): 1-22.

DE LA ROCHE, H.; LETERRIER, J.; GRANDCLAUDE, P.\& MARCHAL, M. 1980. A classification of volcanic and plutonic rocks using R1R2 diagram and major-element analyses - its relationships with current nomenclature. Chem. Geol., 29(3/4): 183-210.

DELHAL, J.; LEDENT, D. \& CORDANI, U. G. 1969. Ages Pb/U; Sr/Rb et $\mathrm{Ar} / \mathrm{K}$ de Formations Métamorphiques et Granitique du Sud-Est du Brésil (Etats de Rio de Janeiro e Minas Gerais). An. Soc Geol. Belg. 92: 271-283.

DUARTE, B., P.; NOGUEIRA, J., R.; HEILBRON, M.\& FIGUEIREDO, M. C. H.1994. Geologia da região de Juiz de Fora e Matias Barbosa (MG). In: CONOR. BRAS. GEOL., 38. Balneáno de Camboriú, 1994. Boletim de Resumos... Balneário de Camboriú, SBG. v. 2, p. 88-90.

EBERT, H. 1957. Relatório de Atividades. In: Relatorio Anual do Diretor da Div. Geol. Min.,DNPM, Rio de Janeiro, Ano 1956: 97-107.

EBERT, H. 1968. Ocorrência da fácies granulítica no sul de Minas Gerais e em áreas adjacentes, em dependência de sua estrutura orogenica: Hipótese sobre sua origem. Anais Acad. Bras. Cienc., 40(supl):215229. Rio de Janeiro.
FIGUEIREDO, M. C. H. \& CAMPOS NETO, M. C. 1993. Geochemistry of charnockitic rocks from Itaperuna region, Rio de Janeiro, Brazil. IN: Congresso de Geoquímica dos Paises de Língua Portuguesa 2, Novembro de 1993, Porto, Portugal

FIGUEIREDO, M.C.H.; CAMPOS NETO, M.C. \& REGO, I.T.S.F. 1989. Geoquímica dos terrenos Juiz de Fora, Paraíba do Sul e Costeiro nos estados do Rio de Janeiro e Espírito Santo. In: Workshop de Geoquímica Isotópica, Geocronologia e Litogeoquímica das Regiões Sul e Sudeste do Brasil, São Paulo, 1989. Boletim de Resumos... SBGqIG/USP, p.41-45.

FYFE, W. S. \& LEONARDOS JR, O. H. 1974. Ancient Metamorphic Migmatite Belts of the Brazilian Atlantic Coast, The African Connection. Rev. Bras. Geoc. 4(4):247-251. São Paulo.

HASUI, Y. \& OLIVEIRA, M. A .F. 1984. A Província Mantiqueira: Setor Central. In: Almeida \& Hasui 1984, O Precambriano do Brasil, Edgard Blucher, $344 \mathrm{p}$.

HEILBRON, M. 1993. Evolução tectono-metamórfica da seção Bom Jardim de Minas-MG - Barra do Pirai-RJ, setor central da Faixa Ribeira. São Paulo. Tese de Doutoramento, IG - Universidade de São Paulo. 268p.

HEILBRON, M. 1995. O Segmento Central da Faixa Ribeira: Sintese Geologica e Ensaio de Evolução Geotectônica. Rio de Janeiro. Tese de Livre Docência, DGEL - Universidade do Estado do Rio de Janeiro. $110 \mathrm{p}$.

IRVINE, T.N. \& BARAGAR, W.R.A. 1971. A guide to the chemical classification of the common volcanic rocks. Canadian Journal of Earth Science, 8:523-548.

JENSEN, L. S..1976. A new cation plot for classifying subalkalic volcanic rocks. Ontario. Ontario Division of Mines, MP 66,22p.

LE MAITRE, R.W. 1989. A Classification of Igneous Rocks and Glossary of Terms. Blackwell, Oxford, $193 \mathrm{p}$

MACHADO FILHO, L.; RIBEIRO, M.; GONZALEZ, S. R.; SCHENINI, C. A.; SANTOS NETO, A. DOS; PALMEIRA, R. C.; PIRES, J. L.; TEIXEIRA, W. \& CẢSTRO, H. E. F. 1983. Geologia das folhas Rio de Janeiro (SF 23/24) escala 1:1.000.000, mapa e texto explicativo. RADAM Brasil-MME, Rio de Janeiro, 780p.

MACHADO, N; VALLADRES, C.S.; HEILBRON, M. \& VALERIANO, C M. $1996 \mathrm{U} / \mathrm{Pb}$ Geochronology of the Central Ribeira belt: Implications for the evolution of Brasiliano Orogeny. Precamb. Res., 79(3-4):347361.

MESCHEDE, M. 1986 A Method of discriminating between different types of mid-ocean ridge basalts and continental tholeiites with the Nb-Zr-Y diagram. Chem. Geol., 56: 207-218.

MULLEN, E,D. 1983. $\mathrm{MnO} / \mathrm{TiO}_{2} / \mathrm{P}_{2} \mathrm{O}_{5}$ : a minor element discriminant for basaltic rocks of oceanic environments and its implications for petrogenesis. Earth Planet. Sci. Let., 274: 53-62.

OLIVEIRA M. A. F. 1980. Petrologia das rochas granuliticas da Faixa Paraiba do Sul, Estados do Rio de Janeiro e Minas Gerais. Tese de Livre Docencia, IGCE-UNESP, 116p (inédito).

OLIVEIRA, M. A. F. 1982. Bulk geochemistry of the Paraíba do Sul Granulitic Belt. Rev. Bras. Geoc., 12(1-3):369-374.

PEARCE, J. A. 1982. Trace element characteristics of lavas at destructive plate boundaries. In: R. S. Thorpe (ed.), Andesites and Related Rocks, Winchester, Wiley, pp. 525-548. 
PEARCE, J. A. 1983. Role of the sub-continental lithosphere in magma genesis at active continental margins. In C J. Hawkesworth and M. J. Norry (editors), Continental Basalts and Mantle Xenoliths. Shiva, Nantwich, pp. 230-249.

PEARCE, J. A. \& CANN, J. R. 1973. Tectonic setting of basic volcanic rocks determined using traces elements. Earth Planetary Science Letters., 19: 290-300.

PEARCE, J. A.; HARRIS, N. B. W. \& TINDLE, A. G. 1984. Trace element driscrimination diagrams for the tectonic interpretation of granitic rocks. Jour. Petrol., 25: 956-983.

PEARCE, J.A. \& NORRY, M.J. 1979. Petrogenetic implications of Ti, Zr, $\mathrm{Y}$ and $\mathrm{Mb}$ variations in volcanic rocks. Contrib. Mineral. Petrol., 69: $33-47$.

ROLLINSON, H. 1993. Using Geochemical Data: Evaluation, presentation, interpretation. Longman Group, England, 3S2p

SAD, J. H. G. \& B ARBOS A, L. M. 1985. A origem dos chamockitos e rochas afins da região do médio Paraíba do Sul, Estado do Rio de Janeiro. IN: Contribuições a Petrologia e a Mineralogia, SBG-Núcleo de Minas Gerais, 1985 p. 15-27.

TAYLOR, S. R. \& McLENNAN, S. M. 1985. The Continental Crust: its Composition and Evolution. Geoscience Texts. Blackwell Sc. Public. Palo Alto, Califórnia. 312 p.
TEIXEIRA, W. \& FIGUEIREDO, M. C. H. 1991. An outline of Early Proterozoic crustal evolution in the São Francisco Craton, Brazil: a review. Precamb. Res., 53:1-22.

TROUW, R.A.J. \& CASTRO, E. M. O. 1996. Significado tectônico dos granulitos de alta pressão no sul de Minas Gerais. In: CONOR. BRAS. GEOL., 39. Salvador, 1996. Anais... Salvador, SBG. v.6:145-148.

VALERIANO, C. M. 1992. Evolução tectônica da extremidade meridional da Faixa Brasília, região da Represa de Furnas, sudoeste de Minas Gerais. Tese de Doutorado, Institute de Geociências. USP, 192 p.

WINCHESTER, J.A. \& FLO YD, P. A. 1976 Geochemical magma type; application to altered metamor-phosed basic igneous rocks. Earth Plannet. Sci. Let., 28: 459-469

WILSON, M. 1988. Igneous Petrogenesis: a global tectonic approach. Unwin Hyman, London 466p

Manuscrito A883

Recebido em 15 de novembro de 1996

Revisao dos autores em 15 de abril de 1997

Revisao aceita em 15 de junho de 1997 